\title{
SPATA33 is an autophagy mediator for cargo selectivity in germline mitophagy
}

\author{
Ying Zhang ${ }^{1,3} \cdot \mathrm{Xu} \mathrm{Xu}^{1} \cdot$ Mengxin $\mathrm{Hu}^{1} \cdot \mathrm{Xin} \mathrm{Wang}^{1} \cdot$ Hanhua Cheng ${ }^{1} \cdot$ Rongjia Zhou ${ }^{1,2}$
}

Received: 23 January 2020 / Revised: 22 September 2020 / Accepted: 6 October 2020 / Published online: 21 October 2020

(c) The Author(s) 2020. This article is published with open access

\begin{abstract}
Selective autophagic degradation of mitochondria (mitophagy) is important in maintaining proper cellular homeostasis. Here, we found that SPATA33 is a novel autophagy mediator for mitophagy in testis. The SPATA33 protein localizes on mitochondria via its binding of the carboxyl terminal with the outer mitochondrial membrane protein VDAC2. Upon starvation induction, SPATA33 is recruited to autophagosome by binding the autophagy machinery ATG16L1 via its Nterminal along with mitochondria. Notably, Spata33 knockout inhibited autophagy and overexpression can promote autophagosome formation for mitochondrial sequestration. Therefore, SPATA33 confers selectivity for mitochondrial degradation and promotes mitophagy in male germline cells.
\end{abstract}

\section{Introduction}

Spermatogenesis is an orderly developmental process, which occurs within seminiferous tubules of the testis. It is a spatio-temporal event whereby undifferentiated spermatogonial germ cells with $2 n$ chromosomes become spermatozoa with half number of chromosomes from spermatogonia, spermatocytes, and spermatids by proliferation, meiosis, and differentiation over a period of several weeks [1]. These processes are highly regulated to maintain cellular homeostasis by renewal and degradation

These authors contributed equally: Ying Zhang, Xu Xu

Edited by E. Baehrecke

Supplementary information The online version of this article (https:// doi.org/10.1038/s41418-020-00638-2) contains supplementary material, which is available to authorized users.

Hanhua Cheng

hhcheng@whu.edu.cn

$\triangle$ Rongjia Zhou

rjzhou@whu.edu.cn

1 Hubei Key Laboratory of Cell Homeostasis, College of Life Sciences, Wuhan, China

2 Renmin Hospital of Wuhan University, Wuhan University, 430072 Wuhan, China

3 Present address: Luoyang Normal University, Henan, China of organelles and macromolecules, in which autophagy plays an important role [2,3].

Autophagy is a cellular process of catabolism within cells, by which undesired cellular organelles and protein aggregates are degraded through autophagosome-lysosome pathway. Mitophagy, as a mitochondrion-specific autophagy, mediates the selective removal of damaged mitochondria [4]. Mitochondria are important organelles that provide energy, regulate programmed cell death and generate reactive oxygen species, and they are also crucial for the functioning of spermatogenesis. Mitophagy, as a cellular protective mechanism, can maintain the quantity and stability of mitochondria. Dysregulations of mitophagy were associated with many human diseases, for example, Parkinson's disease [5], neuroprotection [6], chronic obstructive pulmonary disease [7], cardiac ischemia-reperfusion injury [8], and diabetic kidney disease [9].

Autophagy is active during spermatogenesis. There are some studies demonstrating the effects of autophagy on spermatogenic cells, including spermatogonia stem cells $[10,11]$, spermatogonia [12], spermatocytes [13, 14], and spermatozoa $[15,16]$. Protein profiling of spermatogenic cells has identified several proteins in mice with high homology to the yeast autophagy related gene proteins (ATGs) $[17,18]$. Some of these autophagy related proteins were essential for spermatogenesis. Knockout (KO) of Atg5 and $\operatorname{Atg} 7$ led to loss of testosterone production in Leydig cells in mice [19]. Abnormal acrosome biogenesis in Atg7 and Tbcld20 KO mice [20, 21], meiotic initiation arrest in 
Stra8 KO mice [22], the cytoskeletal disorganization in Sertoli cells in Atg5 and Atg7 KO mice [23], and impaired spermatid differentiation in Atg $7 \mathrm{KO}$ mice [24] have also observed. These mutations eventually caused male infertility.

Several proteins and related pathways in regulation of mitophagy have been identified. The PINK1-PRKN pathway was involved in the regulation of mitophagy for eliminating damaged mitochondria in Parkinson's disease [25]. Within this pathway, mitochondrial protein kinase PINK1 accumulates on damaged mitochondria, recruits and activates PRKN which ubiquitylates mitochondrial proteins. Meanwhile, PRKN activation is also accompanied by its autoubiquitination [26]. Two cytosolic autophagy receptors, NDP52 and OPTN, can recognize ubiquitinated mitochondria via their ubiquitin-binding domains, which also have LIR motif required to bind to LC3B on autophagic membranes [27, 28]. In addition, PINK1-mediated phosphoubiquitin can amplify autophagic signals on damaged mitochondria [28]. These processes eventually lead to mitophagy to clean the damaged mitochondria. Insufficient mitophagy triggers accumulation of damaged mitochondria with stabilized PINK1, which was also associated with disease onset, such as chronic obstructive pulmonary disease pathogenesis [29].

PTEN $\alpha$ was a key factor in cardiac protection via mitochondrial quality control. PTEN $\alpha$ can recruit PRKN onto depolarized mitochondria through protein interaction for mitophagy [30]. Meanwhile, deubiquitinating enzymes can suppress these ubiquitination processes. For example, USP8 can deubiquitinate PRKN [26], while USP30 and USP35 can delay PRKN-mediated mitophagy [31]. Thus, deubiquitination functions as a balancing power in regulation of mitophagy. In addition, there are other receptor proteins that are not directly dependent on PARK2. For example, the mitochondrial E3 ligase MARCH5, but not PRKN, can ubiquitylate and degrade mitophagy receptor FUNDC1 in regulating hypoxia-induced mitophagy [32]. Deficiency of FUNDC1 was also associated with metabolic disorders [33]. However, mitophagy can occur in a ubiquitinindependent manner. In yeast, $\operatorname{Atg} 32$, a protein in the outer mitochondrial membrane, functions as an autophagic receptor through its interaction with Atg8 via its AIM-motif, and with Atg11 via its Atg11-binding domain for mitophagy $[34,35]$. Atg11 acts as a scaffold protein to recruit Atg1 for autophagy initiation [36]. Although lack of Atg32 in mammals, the outer mitochondrial membrane proteins, BCL2L13 [37], BNIP3 [38], BNIP3L/NIX [39, 40], and FKBP8 [41], FUNDC1 [42], as well as inner mitochondrial membrane protein, PHB2 [43, 44], serve as functions of autophagy receptors similar to Atg32.

In addition to degradation of damaged mitochondria, elimination of needless or nondamaged mitochondria occurs as a critical quantity control mechanism for maintaining the proper amount of mitochondria [45]. Paternal mitochondria removal in zygote is a key step to ensure maternal inheritance of mitochondria. Both ubiquitin proteasome system and sperm mitophagy occurred during the elimination process [46-49]. Studies in the porcine zygote suggested that a combined action of SQSTM1-dependent autophagy and VCP-mediated ubiquitination of sperm mitochondrial proteins was responsible for sperm mitophagy [47, 48]. In Caenorhabditis elegans, LC3 was a key regulator to control the fate of sperm mitochondria via autophagosome targeted to the pericentrosomal area [50]. Recent study showed that FNDC-1 was a mitophagy receptor and essential for paternal mitochondria elimination in C. elegans [51].

Despite these mitophagy receptors being characterized, tissue or cell-type specific receptors for mitophagy and their precise mechanisms of recognition and degradation are still unclear. We have previously identified a novel gene Spata33, also called as 4732415M23Rik or C16orf55, which is conserved in mammals and specifically expressed in mouse testis [52]. Pathological analysis showed that SPATA33 was decreased in testis of spermatozoa deficient patients [53]. In this study, we report that SPATA33 functions as autophagy mediator as well as its roles in promoting mitophagy in germline. SPATA33 is a novel autophagy mediator for mitophagy through directly binding to the autophagy machinery ATG16L1 and the outer mitochondrial membrane protein VDAC2. This characteristic confers the cargo selectivity during mitophagy.

\section{Results}

\section{SPATA33 is colocalized with autophagy proteins associated with mitochondria of mouse germline cells}

Our previous study indicated that the novel gene Spata33 is evolutionarily conserved in mammals and associated with spermatogenesis [52]. In expression timing, SP1 precedes SPATA33 in postnatal testis, and it can specifically bind to the Spata33 promoter region in vivo and activates its expression in testis (Fig. S1). To further explore functions of SPATA33 in the germline, we first analyzed its expression relationship with potential partners. Immunofluorescence analysis of SPATA33 protein in adult mouse testis showed a co-expression pattern with key autophagy protein ATG16L1 in the cytoplasm of three main types of cells, including Sertoli, Leydig, and spermatogenic cells (Fig. 1a). Cell sorting of testis samples and RT-PCR confirmed Spata33 expression in spermatogonia, spermatocytes, round spermatids, and spermatozoa (Fig. 1b, c). Further sperm immunofluorescence indicated that 

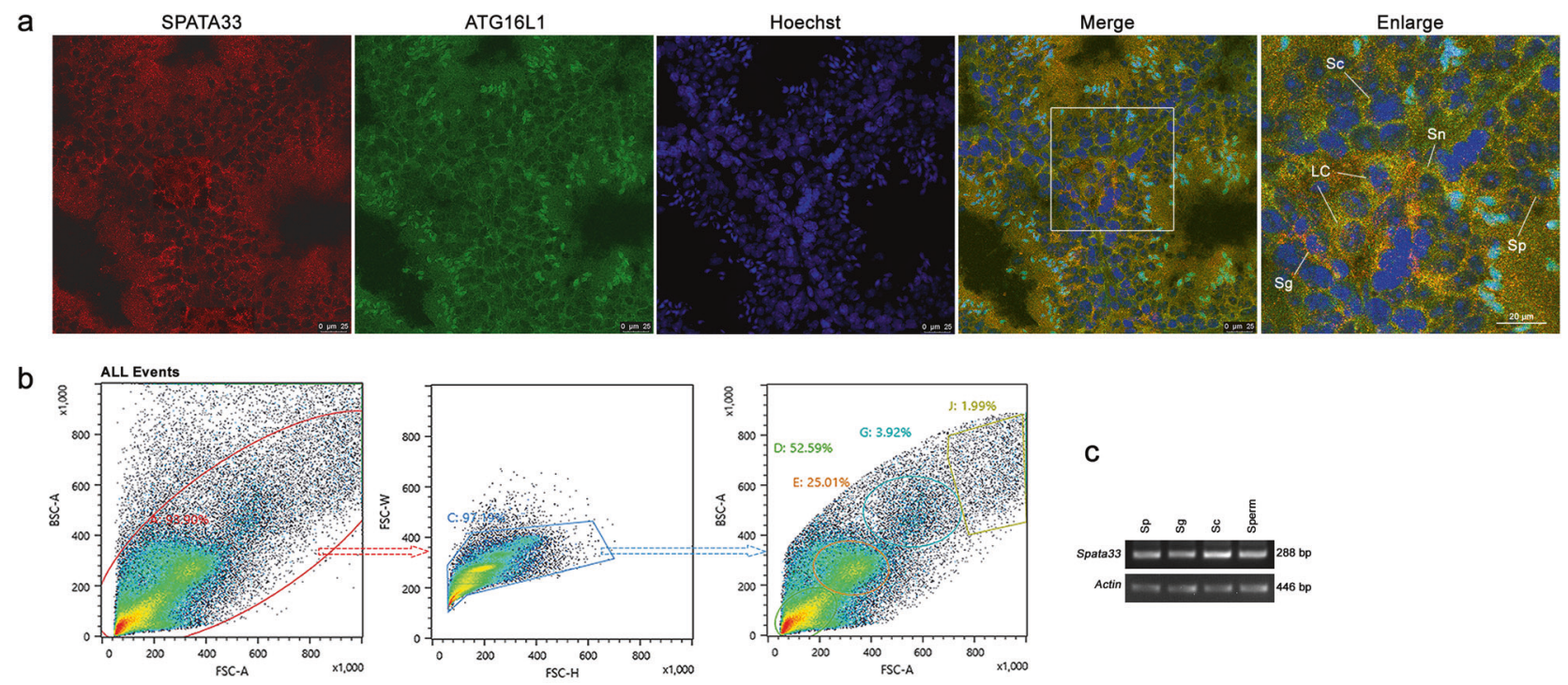

C
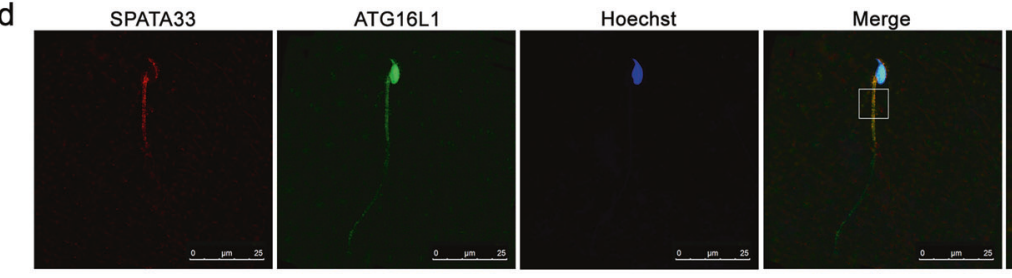

Enlarge
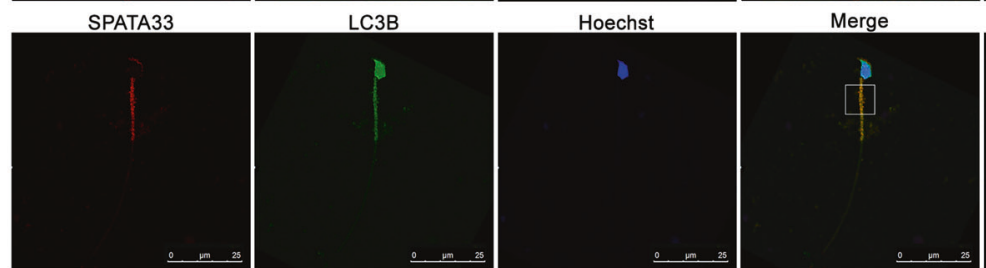

SPATA33

VDAC2

Hoechst

Merge
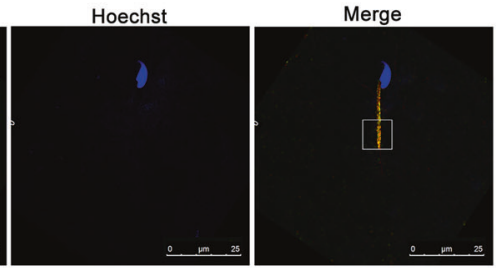

Enlarge
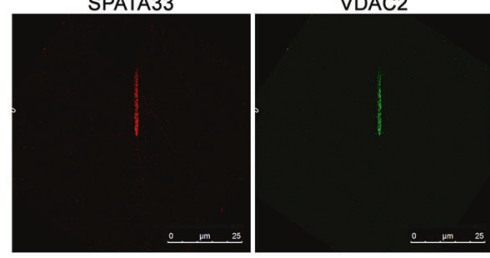

Hoechst
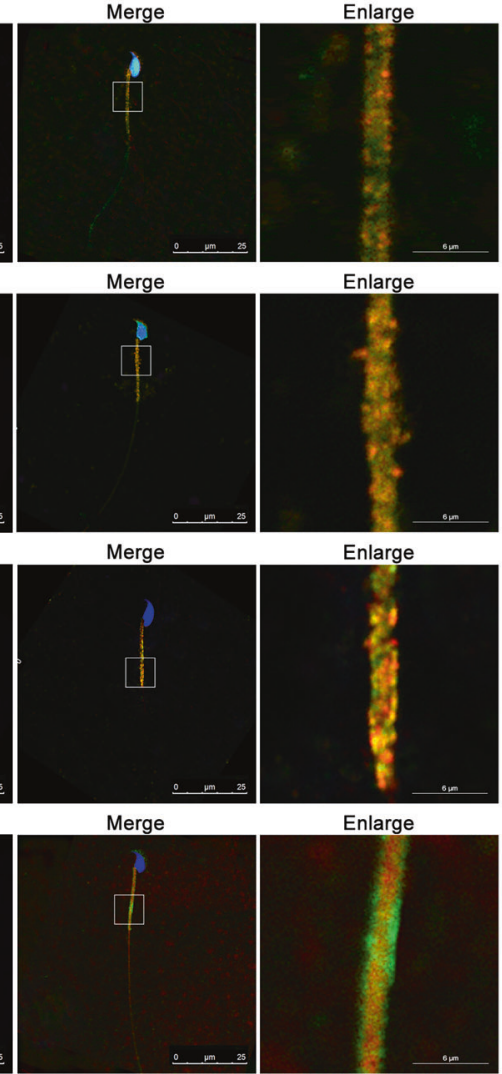

Enlarge

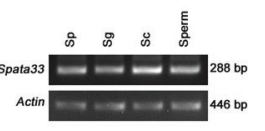

SPATA33
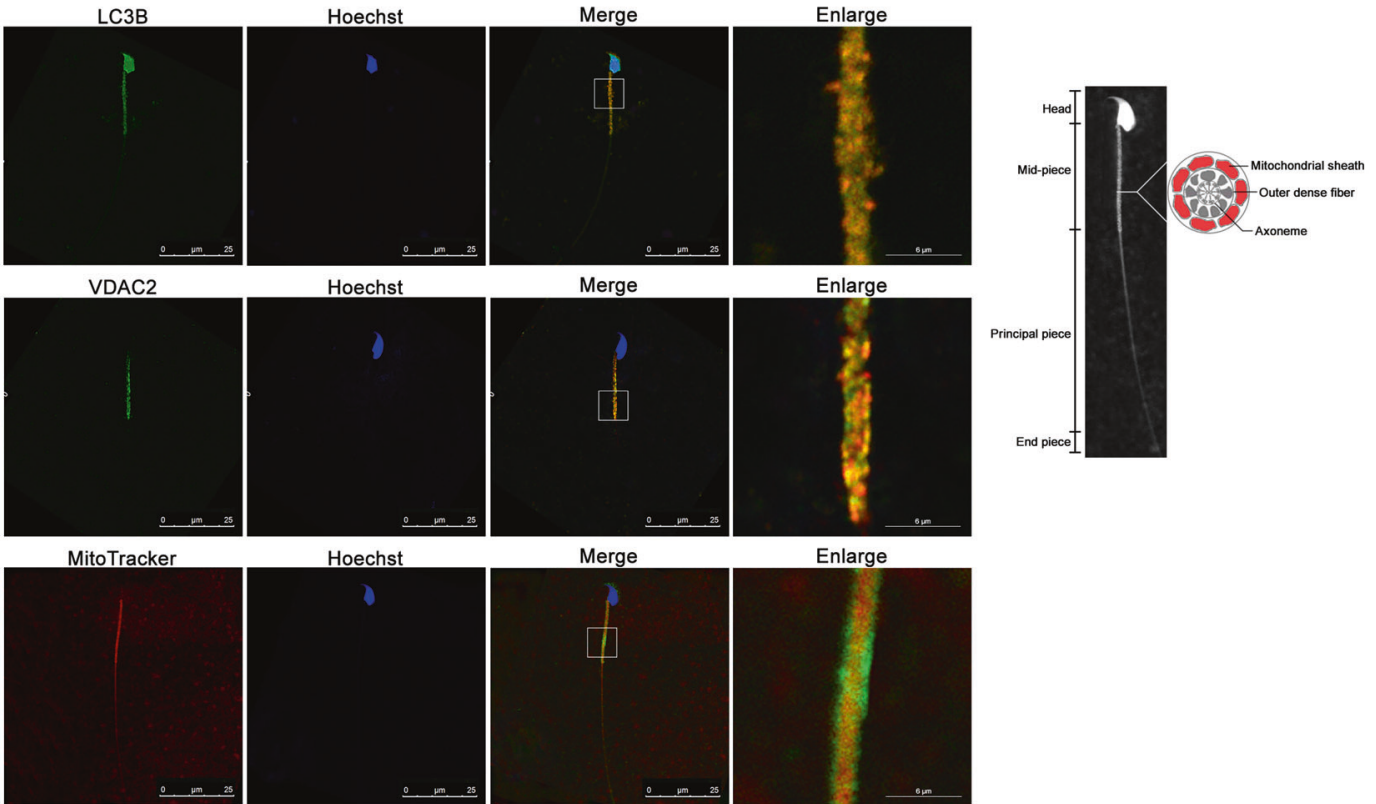

SPATA33 protein was colocalized with key autophagy proteins ATG16L1 and LC3B, and mitochondrial outer membrane protein VDAC2 in the mid-piece region (mitochondria region) of spermatozoa from epididymis of adult mice (Fig. 1d). In addition, MitoTracker (a mitochondria matrix marker) co-staining indicated that SPATA33 protein was located in the mitochondrial sheath of the mid-piece region (Fig. 1d). These results suggested that SPATA33 is potentially associated with mitophagy in spermatogenesis.

\section{SPATA33 is associated with autophagy through interaction with ATG16L1}

To investigate potential molecular mechanisms of SPATA33 in autophagy, coimmunoprecipitation analysis was used to determine interaction of SPATA33 with ATG16L1. Coimmunoprecipitation with either anti-MYC or anti-FLAG after co-transfection of MYC-SPATA33 and FLAG-CherryATG16L1 showed that SPATA33 can interact with 
Fig. 1 SPATA33 and ATG16L1 are colocalized in germline cells in mice. a Immunofluorescence of SPATA33 and ATG16L1 proteins in mouse testis. Anti-SPATA33, anti-ATG16L1 (Alexa Fluor 488 ), and TRITC-conjugated goat anti-rabbit $\operatorname{IgG}(\mathrm{H}+\mathrm{L})$ antibodies were used to detect SPATA33 (red) and ATG16L1 (green). The nuclei were stained by Hoechst (blue). Images were taken by confocal fluorescence microscopy (SP8, Leica, Wetzlar, Germany). Positive signals were detected in Leydig cells (LC), Sertoli cells (Sn), spermatogonia (Sg), spermatocytes (Sc), and spermatids (Sp). Scale bar, $25 \mu \mathrm{m}$; scale bar in enlarged panels: $20 \mu \mathrm{m}$. b Cell sorting of spermatogonia (Sg), spermatocytes (Sc), and the round spermatids (Sp) from testis by cell flow sorter. Serial charts of cells were indicated. c Expression of Spata33 was detected in spermatogonia $(\mathrm{Sg})$, spermatocyte $(\mathrm{Sc})$, the round spermatids (Sp), and spermatozoa (sperm). The sperm cells were isolated from epididymis of adult mouse. RT-PCR was performed from mRNAs isolated from these cells. Actin was used as an internal control. d SPATA33 was colocalized with ATG16L1, LC3B, and VDAC2 in the mid-piece region (mitochondria region) of the sperm cells. The spermatozoa were extracted from epididymis of adult mice. The cells were stained with MitoTracker (mitochondria matrix marker, red). Immunofluorescence analysis were performed with anti-SPATA33, antiATG16L1 (Alexa Fluor 488), anti-VDAC2, anti-LC3B (Alexa Fluor 488), TRITC-conjugated goat anti-rabbit IgG $(\mathrm{H}+\mathrm{L})$, and FITCconjugated rabbit anti-goat $\operatorname{IgG}(\mathrm{H}+\mathrm{L})$ antibodies. The enlarged images were originated from the squares in the merged panels. The nuclei were stained by Hoechst (blue). Images were taken by confocal fluorescence microscopy (SP8, Leica). The graph on the right indicates sperm ultrastructure, highlighting cross-section of the mid-piece region, including axoneme, outer dense fiber, and mitochondrial sheath (red). Scale bar: $25 \mu \mathrm{m}$; scale bar in enlarged panels: $6 \mu \mathrm{m}$.

ATG16L1 (Fig. 2a). Deletion analysis indicated that SPATA33 was bound to the C-terminus of ATG16L1, but not to the N-terminus of ATG16L1 (Fig. 2b). Coimmunoprecipitation experiment confirmed that SPATA33 can interact with endogenous ATG16L1 (Fig. 2c). Further fluorescence microscopy analysis showed that both SPATA33 and ATG16L1 were colocalized in HeLa cells, upon starvation induction in particular (Fig. 2d). Statistical analysis showed that number of colocalized puncta between SPATA33 and ATG16L1 was significantly higher under starvation condition in comparison with normal culture (Fig. 2e). Furthermore, SPATA33 had also obvious colocalization with key autophagy protein LC3B (Fig. 2f, g). These results indicated that SPATA33 was associated with autophagy through its interaction with ATG16L1.

\section{Spata33 knockout suppresses mitophagy}

To further explore roles of SPATA33 in mitophagy, we constructed both Spata33 ${ }^{-1-}$ TM4 (Sertoli cells) and GC-1 (spermatogenic cells) cell lines using CRISPR/Cas9 technology. Sequencing and off-target analysis showed that Spata33 was efficiently KO in both cell lines (Figs. S2-S5). Spata33 $^{-1-}$ cells (\#32-10) were further used to detect autophagosome formation under starvation condition. Immunofluorescence analysis showed that obvious LC3B puncta were detected at $1 \mathrm{~h}$ under starvation culture condition in $\mathrm{Spata33}^{-/-}$cells, whereas LC3B puncta appeared at $0.5 \mathrm{~h}$ under starvation condition in wild-type cells (Fig. 3a, b, e). When Spata33 expression was rescued in Spata33 $3^{-1}$ cells by infecting with lentivirus expressing Spata33, obvious LC3B puncta appeared again at $0.5 \mathrm{~h}$ under starvation condition (Fig. 3c, e). In Spata33 overexpression in WT cells by infecting with lentivirus expressing Spata33, LC3B puncta were also observed at $0.5 \mathrm{~h}$ under starvation condition (Fig. 3d, e). These results showed that Spata33 overexpression promoted formation of autophagosome, while Spata33 KO inhibited the formation of autophagosome. Western blot analysis showed that LC3B-II, which is a lipidated form of the key autophagy protein LC3B-I, was significantly decreased in Spata33 ${ }^{-1-}$ cells upon starvation induction in comparison with the normal culture condition (Fig. 4a). SQSTM1, as a substrate for autophagy degradation, had an opposite trend (Fig. 4a). At the same time, we also examined whether protein levels of mitochondrial outer membrane VDAC2 and inner membrane COX-IV were affected when autophagy decreased. Western blot analysis showed that starvation treatment resulted in an obvious decrease of degradation of VDAC2 and COX-IV in Spata33 KO compared to wild type, indicating that mitochondria was an autophagic target (Fig. 4a). The Atg16l1 gene KO cell lines were also constructed, and LC3B-II, SQSTM1, VDAC2, and COX-IV were detected by the same method. It was found that the Atg16l1 KO showed a similar effect on autophagy inhibition as Spata33 KO (Fig. 4b).

In addition, we observed damaged mitochondria being engulfed by autophagosomes in Spata33-overexpressed GC1 cell line by transmission electron microscope under combined treatment of starvation and CCCP (an inducer of mitophagy), in addition to autolysosomes, in which damaged mitochondria were being degraded (Fig. 4c). Statistical analysis showed that the level of mitochondrial autophagy in Spata33-overexpressed cells was significantly higher than that in wild-type cells (Fig. 4c). Furthermore, MitoQC-based analysis using the pmCherry-GFP-FIS $1^{101-152}$ tandem reporter (FIS1, a mitochondrial outer membrane protein) in Spata33 KO (Spata33 ${ }^{-1-}$,\#33), Spata33 overexpression, and wild-type GC-1 cell lines showed that Spata33 KO significantly decreased the level of mitophagy, while Spata33 overexpression promoted mitophagy (Fig. 5a-f). Together, these results suggested that SPATA33 promoted mitophagy in germ cells.

\section{SPATA33-associated autophagy flux}

To investigate SPATA33-associated autophagy process, we tested the SPATA33-involved autophagy flux through Spata33 KO and forced expression. Autophagy flux tests were performed using a tandem fluorescent indicator, 

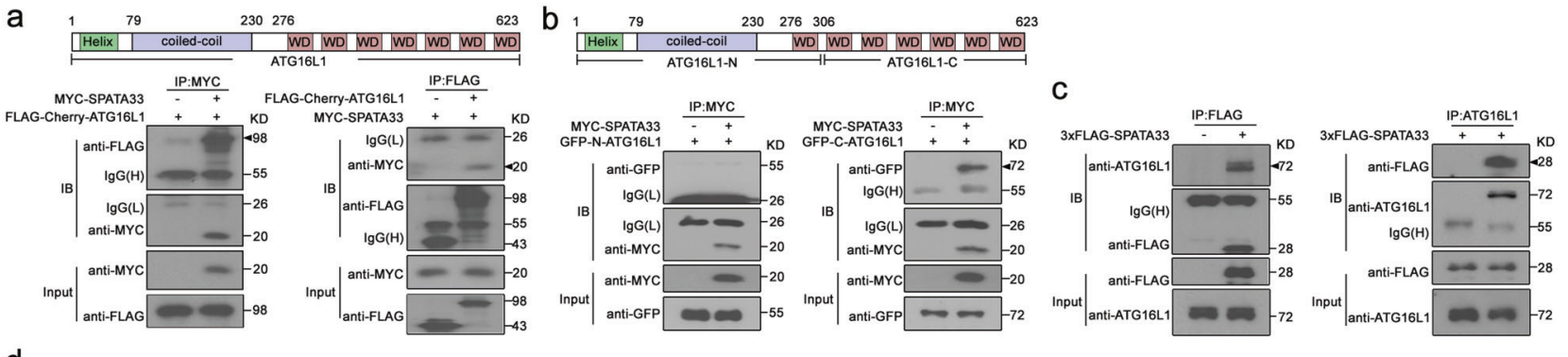

d

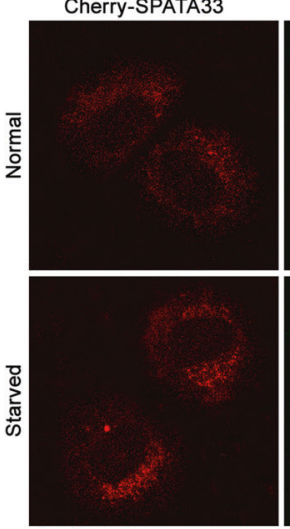

GFP-ATG16L1
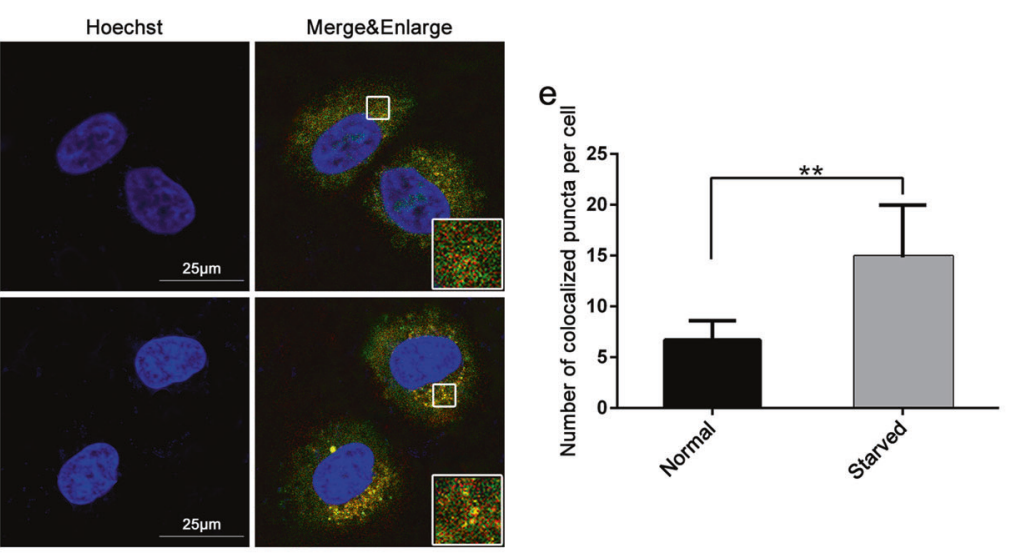

f

Cherry-SPATA33
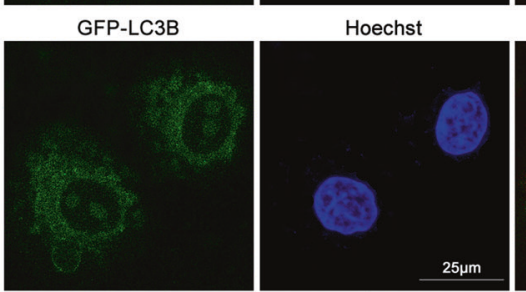

Merge\&Enlarge
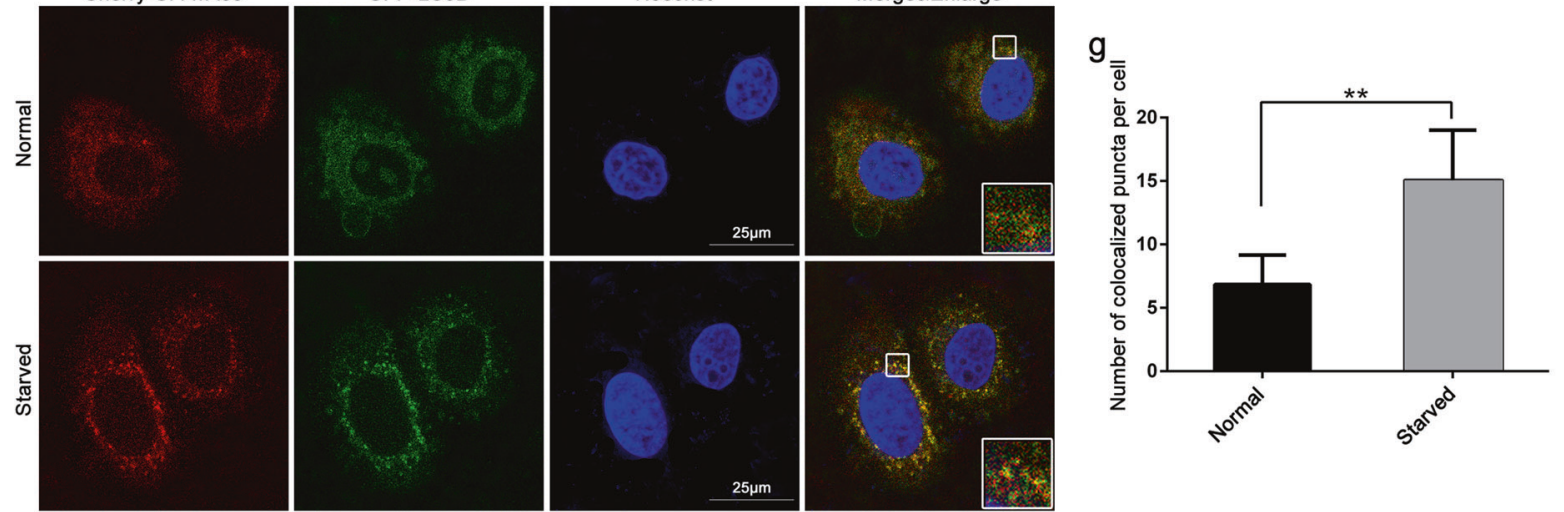

Fig. 2 SPATA33 interacts with ATG16L1 to form autophagic puncta upon starvation induction. a Coimmunoprecipitation of MYC-SPATA33 with Cherry-FLAG-ATG16L1. HEK293T cells were transiently transfected with pMYC-SPATA33 and pCherry-FLAGATG16L1. After $48 \mathrm{~h}$, the whole cell lysate was extracted for coimmunoprecipitation with anti-MYC, or anti-FLAG. Anti-FLAG or antiMYC was also used for western blotting. Arrowheads indicate the immunoprecipitated bands. b Coimmunoprecipitation between MYC-SPATA33 and deletion mutants of ATG16L1. pMYCSPATA33 was transiently co-transfected with pGFP-N-ATG16L1 or pGFP-C-ATG16L1 in HEK293T cells. Cell lysates were examined by western blotting using the anti-MYC or anti-GFP antibody. For coimmunoprecipitation, the lysates were immunoprecipitated with anti-MYC, followed by immunoblotting with the anti-GFP antibody. Arrowheads indicate the immunoprecipitated bands. c Endogenous ATG16L1 interacted with FLAG-SPATA33. HEK293T cells were transiently transfected with p3xFLAG-SPATA33, and after $48 \mathrm{~h}$, the whole cell lysate was extracted for coimmunoprecipitation with antiATG16L1 or anti-FLAG. Anti-ATG16L1 or anti-FLAG antibody was

also used for western blotting. d Colocalization of SPATA33 with ATG16L1 in HeLa cells under starvation condition. HeLa cells were transiently co-transfected with pCherry-SPATA33 and pGFPATG16L1. After cultured in normal medium for $24 \mathrm{~h}$, the cells were starved in EBSS medium for $2 \mathrm{~h}$, and analyzed by confocal microscopy. The nuclei were stained by Hoechst (blue). The insets showed an enlarged view of the indicated squares. Yellow puncta in merged panels are the colocalized puncta. Scale bar: $25 \mu \mathrm{m}$. e Statistical analysis of colocalized puncta between SPATA33 and ATG16L1. f Colocalization of SPATA33 with LC3B in HeLa cells under starvation condition. HeLa cells were transiently co-transfected with pCherry-SPATA33 and pGFP-LC3B and cultured in normal medium for $24 \mathrm{~h}$. The cells were starved in EBSS medium for $2 \mathrm{~h}$, and analyzed by confocal microscopy. The nuclei were stained by Hoechst (blue). The insets showed an enlarged view of the indicated squares. Yellow puncta in merged panel are the colocalized puncta. Scale bar: $25 \mu \mathrm{m}$. g Statistical analysis of colocalized puncta between SPATA33 and LC3B. Data are presented as means \pm S.D. $* * p<0.01(n=3$ independent experiments). 

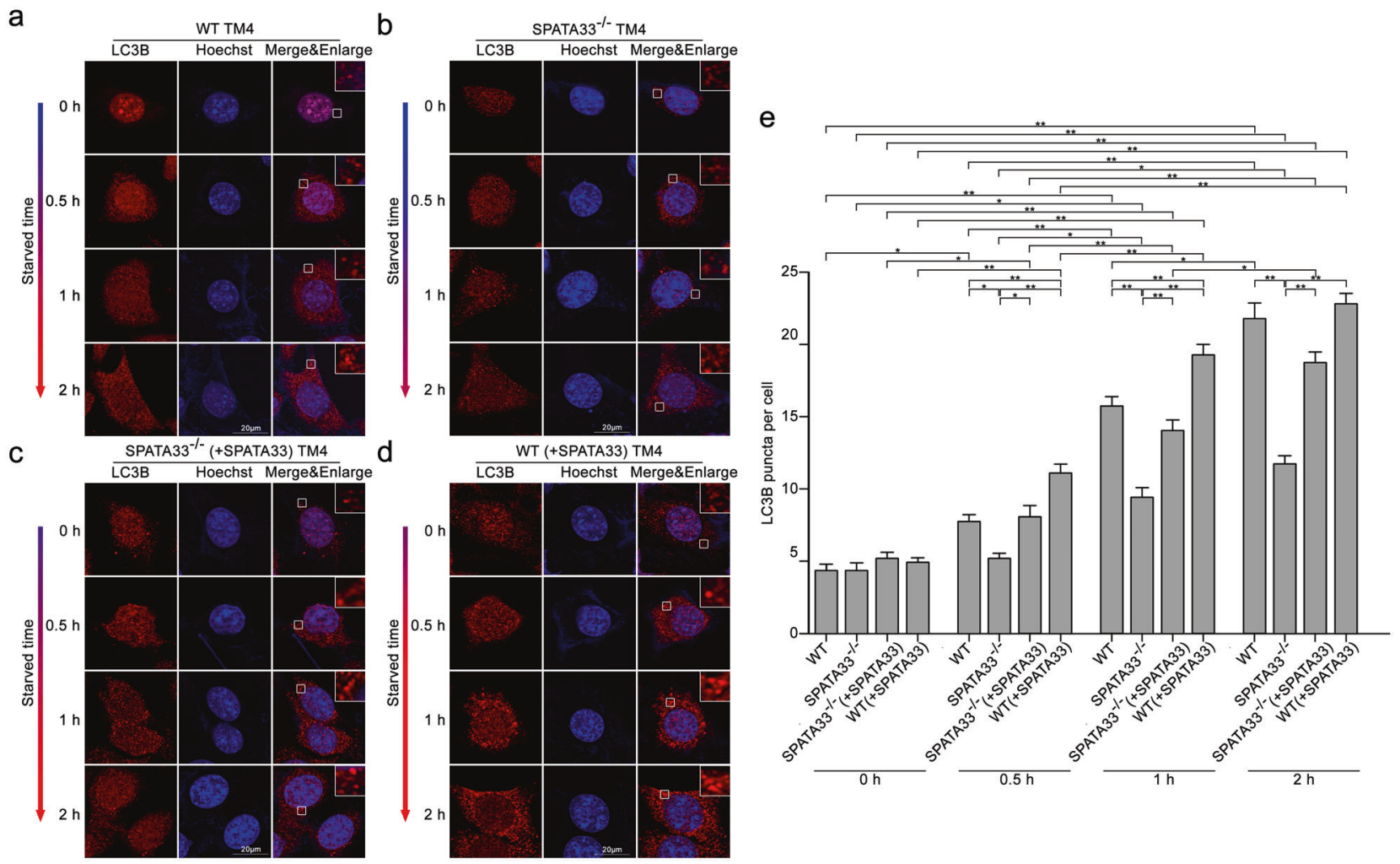

Fig. 3 Spata33 knockout inhibits autophagy. a LC3B puncta were detected in WT TM4 cells after starvation culture (EBSS) for $0.5 \mathrm{~h}$. The cells were cultured in EBSS for the indicated time and analyzed by immunofluorescence with the anti-LC3B antibody and confocal microscopy. The insets showed an enlarged view of the indicated squares and highlighted red LC3B puncta in the cytoplasm. The nuclei were stained with Hoechst reagent. Scale bar: $20 \mu \mathrm{m}$. b LC3B puncta were detected at 1 and $2 \mathrm{~h}$ under starvation culture in the Spata33 $3^{-/-}$ cells. Scale bar: $20 \mu \mathrm{m}$. c To rescue Spata33 expression in the Spata $33^{-/-}$cells, the cells were infected with lentivirus expressing

3xFLAG-SPATA33. Immunofluorescence with the anti-LC3B antibody showed obvious LC3B puncta appeared again at $0.5 \mathrm{~h}$ under starvation culture. Scale bar: $20 \mu \mathrm{m}$. d To overexpress Spata33 in the WT TM4 cells, the cells were infected with lentivirus expressing $3 \times$ FLAG-SPATA33 and analyzed by immunofluorescence with the anti-LC3B antibody. Obvious LC3B puncta were detected again at $0.5 \mathrm{~h}$ under starvation culture. Scale bar: $20 \mu \mathrm{m}$. e. Statistical analysis of LC3B puncta from (a) to (d). The number of LC3B puncta in 30 cells was counted for each group. Data are presented as mean \pm S.D. $* p<0.05 ; * * p<0.01$ ( $n=3$ independent experiments).

mCherry-GFP-LC3B, in Spata33 KO (Spata33 ${ }^{-1-}$, \#33), SPATA33 rescued (stably expressing SPATA33 in

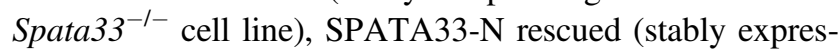
sing SPATA33-N in Spata33 ${ }^{-1-}$ cell lines), SPATA33-C rescued (stably expressing SPATA33-C in Spata33 ${ }^{-1-}$ cell lines), and wild-type GC-1 cell lines. Since green fluorescence of the fusion protein is very sensitive to the acidic environment of lysosomes and quickly quenched in autolysosomes, just red fluorescence could be detected in autolysosomes. Fluorescence analysis using the tandem fluorescent indicator system in these cell lines showed that Spata33 KO significantly inhibited formation of autophagosomes, while both rescues of SPATA33 and SPATA33-N promoted the formation of autophagosomes (Fig. 6a-d, i). Under normal conditions or bafilomycin A1 treatment, there was no significant difference between these cell lines (Fig. 6a, b, e, f). However, SPATA33-N rescue could promote the formation of autophagosome, while SPATA33$\mathrm{C}$ could not rescue autophagy inhibition under starvation condition (Fig. 6c, g). This also confirmed that the interaction between SPATA33 and ATG16L1 was necessary to promote autophagy. Further starvation and bafilomycin A1 combined treatment showed a significant accumulation of autophagosomes (Fig. 6d, h). These results suggested that SPATA33 plays a role in autophagosome formation.

\section{SPATA33 mediates mitophagy through linking between VDAC2 and ATG16L1}

To further explore how SPATA33 mediates mitophagy, we analyzed SPATA33 interaction with the mitochondrial outer membrane protein VDAC2 by coimmunoprecipitation. We found that SPATA33 can interact with VDAC2 in the coimmunoprecipitation analysis (Fig. 7a). SPATA33 interacted via its C-terminus with VDAC2, but not to its $\mathrm{N}$-terminus (Fig. 7a, b). Deletion mapping and coimmunoprecipitation showed that ATG16L1 bound to the N-terminus of SPATA33 (Fig. 7c, d), whereas VDAC2 


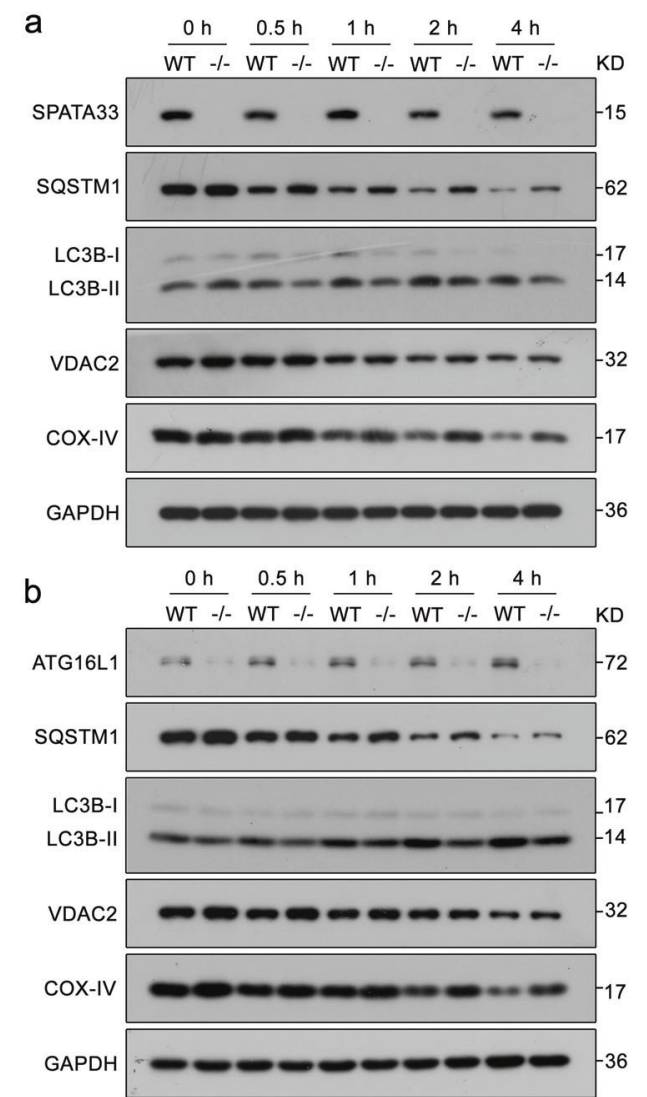

c

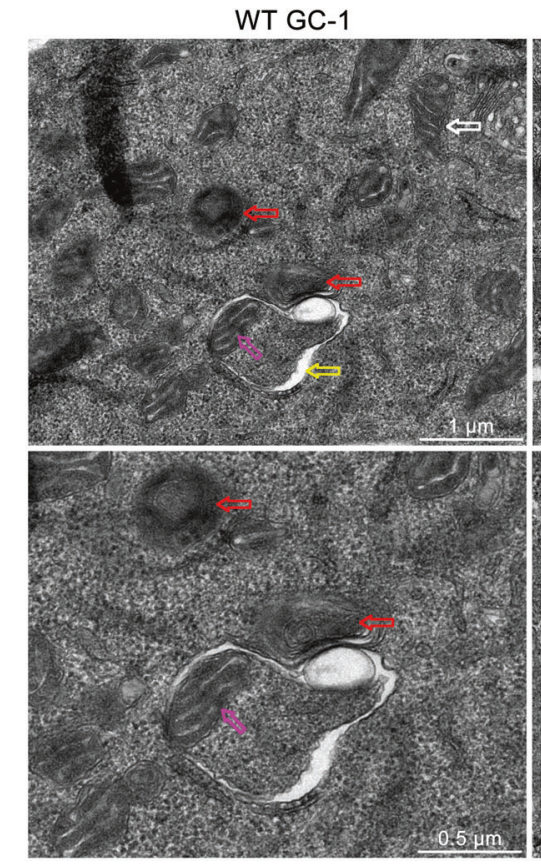

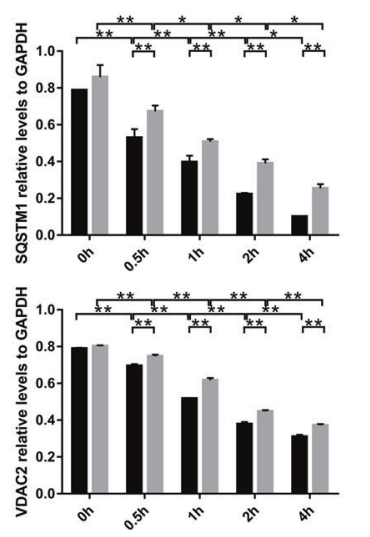
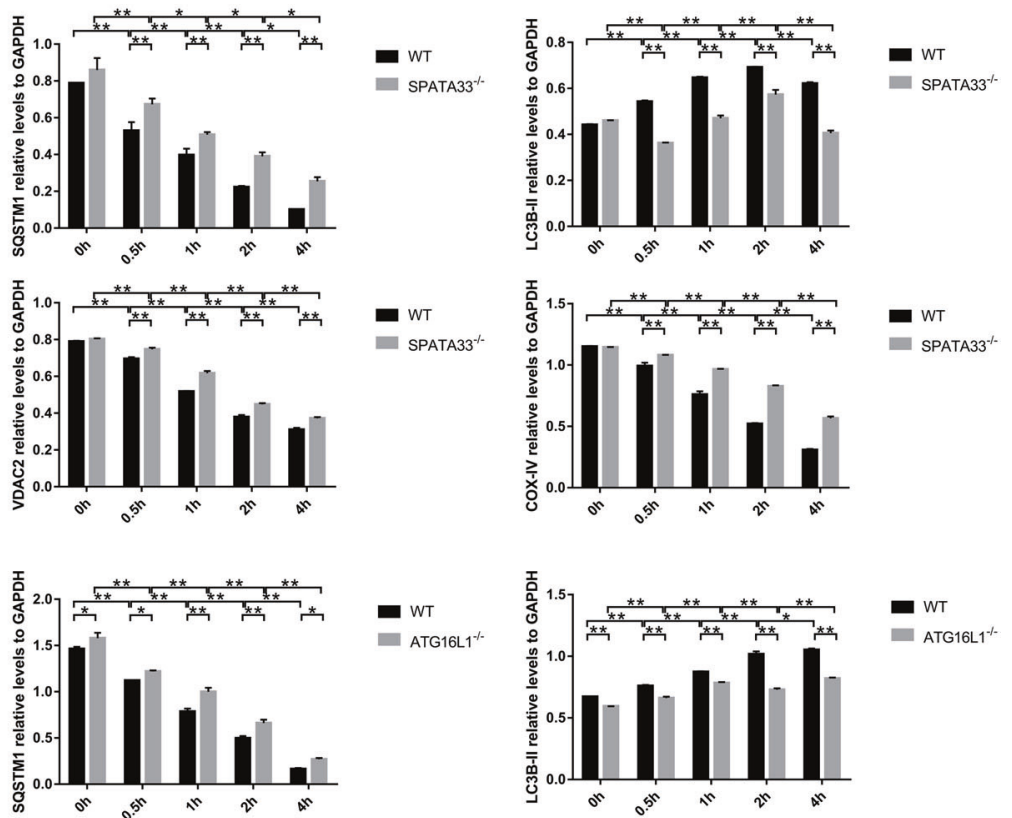

WT
ATG16L $1^{-/}$
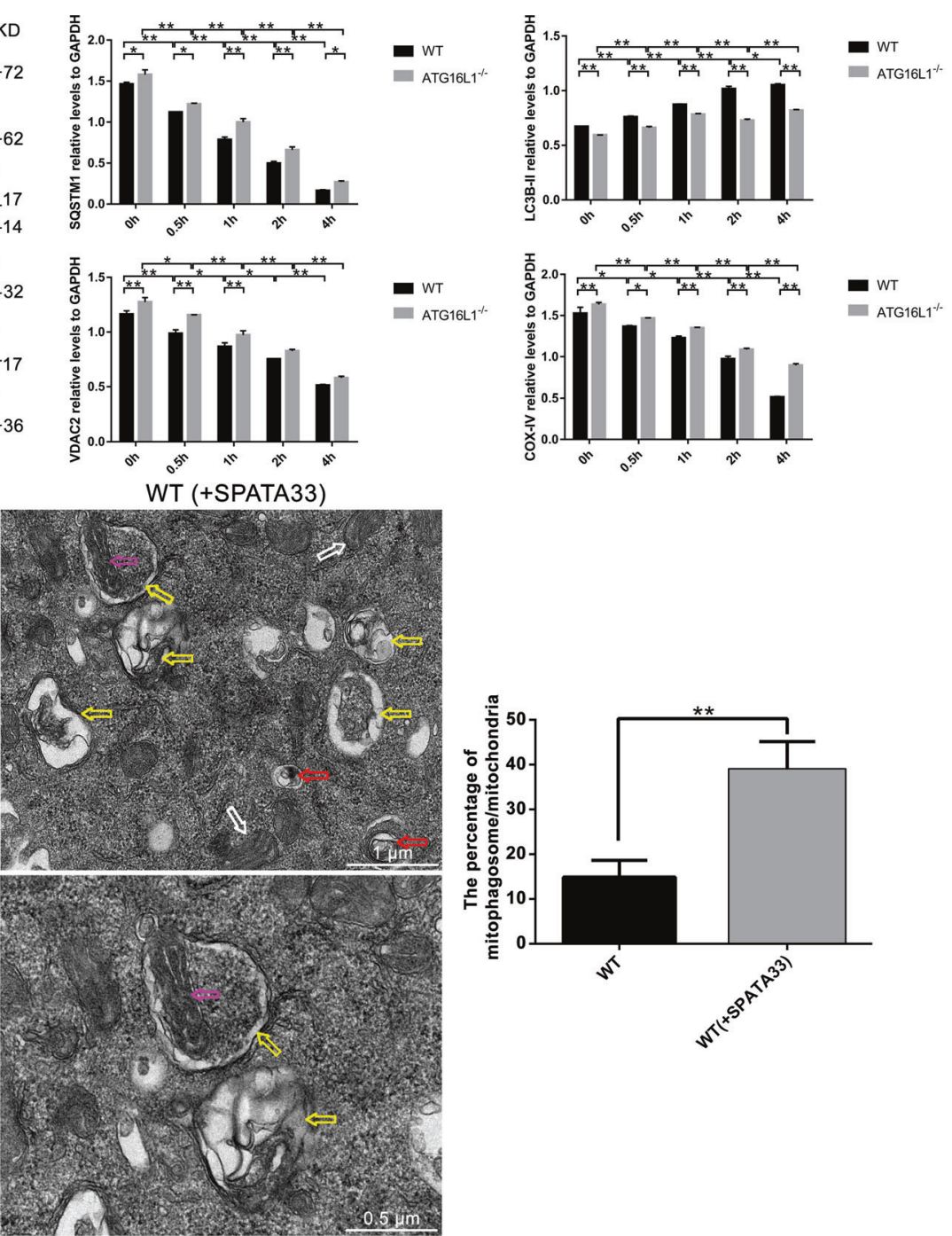

interacted with the C-terminus of SPATA33. Endogenous interactions among SPATA33 and ATG16L1 or VDAC2 were confirmed in GC-1 cell lines (Fig. 7e). Fluorescence microscopy showed that there was an obvious colocalization between Cherry-SPATA33 and GFP-VDAC2 and
ATG16L1, which were enhanced significantly by starvation and CCCP combined treatment (Fig. 7f, g). A similar result was also observed in HeLa cells (Fig. S6). Taken together, SPATA33 mediates mitophagy via its carboxyl terminal by interaction with the mitochondrial outer membrane protein 
Fig. 4 Spata33 knockout inhibits mitophagy. a Spata33 knockout decreased autophagy and mitochondrial related protein levels. WT TM4 and Spata33 ${ }^{-1-}$ cell lines (\#32-10) were cultured in the EBSS medium for $0,0.5,1,2$, and $4 \mathrm{~h}$, respectively. Cell lysates were analyzed by immunoblotting with the anti-SQSTM1, anti-LC3B, antiVDAC2, or anti-COX-IV. GAPDH was used as an endogenous control. The graphs on the right panels indicate statistical analysis of the gray scanned SQSTM1, LC3B, VDAC2, and COX-IV from (a). b Atg16l1 knockout decreased autophagy and mitochondrial related protein levels. WT TM4 and Atg16l1 ${ }^{-1-}$ cell lines (\#1-13, the group were not from single KO clone) were cultured in the EBSS medium for $0,0.5,1,2$, and $4 \mathrm{~h}$, respectively. Cell lysates were analyzed by immunoblotting with the anti-SQSTM1, anti-LC3B, anti-VDAC2, or anti-COX-IV antibody. GAPDH was used as an endogenous control. The graphs on the right panels indicate statistical analysis of the gray scanned SQSTM1, LC3B, VDAC2, and COX-IV from (b). c Electron microscopy showed the mitophagosome in wild type and SPATA33overexpressed GC-1 cells. Enlarged images were showed in the lower panels. The yellow arrow indicates autolysosome, the purple arrows show the degraded mitochondria in autolysosome, the white arrow indicates the normal mitochondria, and the red arrow indicates lysosome. Scale bar: $1 \mu \mathrm{m}$, scale bar in enlarged panels: $0.5 \mu \mathrm{m}$. The graphs on the right indicate statistical analysis of the percentage of mitophagosome/all mitochondria. Data are presented as means \pm S.D. $* * p<0.01$ ( $n=3$ independent experiments).

VDAC2, while SPATA33 can also interact via its Nterminal with the WD40 region of ATG16L1 protein (Fig. 7h). Upon starvation stress, damaged mitochondria are encapsulated by pre-autophagosome through mediator SPATA33 linking between VDAC2 and ATG16L1 to initiate mitophagy.

\section{Discussion}

Selective autophagy is highly regulated through cargo selectivity and autophagy receptors, relative to formerly nonselective bulk degradation pathway. Mitophagy, as a typic process of selective autophagic degradation of mitochondria, plays important roles in cellular quality control that eliminates damaged and superfluous mitochondria. Dysregulation of mitophagy has been associated with several human diseases, including neurodegenerative disorders such as Parkinson's disease [54]. Despite the considerable efforts in identification of autophagy receptors and pathways involved in mitophagy, tissue or cell-type specific receptors for mitophagy and their precise mechanisms of recognition and degradation are still unclear. Here we identify SPATA33 as a novel autophagy mediator for mitophagy in germline. The autophagy mediator is particularly important, as it not only broadens our understanding of cellular quality control and mitochondrial homeostasis, but also has the implications of the SPATA33-mediated mitophagy in the germline development and diseases.
The autophagy mediator SPATA33 has several distinct features in mammalian mitophagy. In autophagy functions, it can promote mitophagy through interaction with ATG16L1 upon stress, such as starvation and mitochondrial damage, as well as being an autophagy mediator. Autophagy flux assays confirmed that it promoted the formation of autophagosomes, but not influenced autophagosome fusion with lysosome. In yeast, mitophagy is mediated by the outer mitochondrial membrane receptor Atg32, which links mitochondria to the phagophore by directly binding Atg8 via its AIM-motif (Atg8-interacting motif) [34]. Atg32 can also interact with scaffold protein Atg11, which eventually recruits Atg1 complexes to induce autophagy [36]. However, a counterpart to yeast Atg11 has yet to be discovered in mammals. In this study, we suggest that SPATA33 may exert roles as a bi-functional counterpart of both $\operatorname{Atg} 32$ and $\operatorname{Atg} 11$ in mammals. The difference is that SPATA33 can directly links damaged mitochondria to autophagosomes via its binding to the outer mitochondrial membrane protein VDAC2, as well as to key autophagy machinery ATG16L1. This characteristic probably confers an efficient and specific mechanism of cellular quality control through SPATA33-mediated mitophagy in mammalian germline.

In SPATA33-mediated mitophagy, cargo selectivity is provided by the autophagy protein SPATA33, which directly links targeted mitochondria via its binding to the outer mitochondrial membrane protein VDAC2 and to the autophagic machinery ATG16L1. The mitophagy process is probably ubiquitin independent. So far, all ubiquitinindependent autophagy receptors on the outer mitochondrial membrane (BCL2L13, BNIP3, NIX, FKBP8, FUNDC1, and Atg32) have a common LIR/AIM-motif, which can bind to LC3B/Atg8 on autophagic membranes [55]. Compared with these receptors, SPATA33 directly interacts with the autophagic machinery ATG16L1, instead of LC3B. Thus, SPATA33-mediated mitophagy probably occurs when ATG5-ATG12 recruits ATG16L1 to form ATG5ATG12-ATG16L1 complex on autophagic membranes upon autophagy induction. In autophagy vesicle elongation, ATG12-ATG5-ATG16L1 complex assembly is a key event. The small GTPase RAB37 regulates autophagosome formation through a switch between the GTP-bound "on" and the GDP-bound "off" forms. RAB37-GTP interacts directly with ATG5 and promotes the interaction of ATG12-ATG5 with ATG16L1 [56, 57]. The RAB37-ATG12-ATG5ATG16L1 complex eventually recruits and lipidates LC3B-I to form active LC3B-II, which accelerates autophagosome formation.

Tissue or cell-type specific mitophagy is important to degrade unnecessary or damaged mitochondria to maintain mitochondrial homeostasis in development and diseases. 

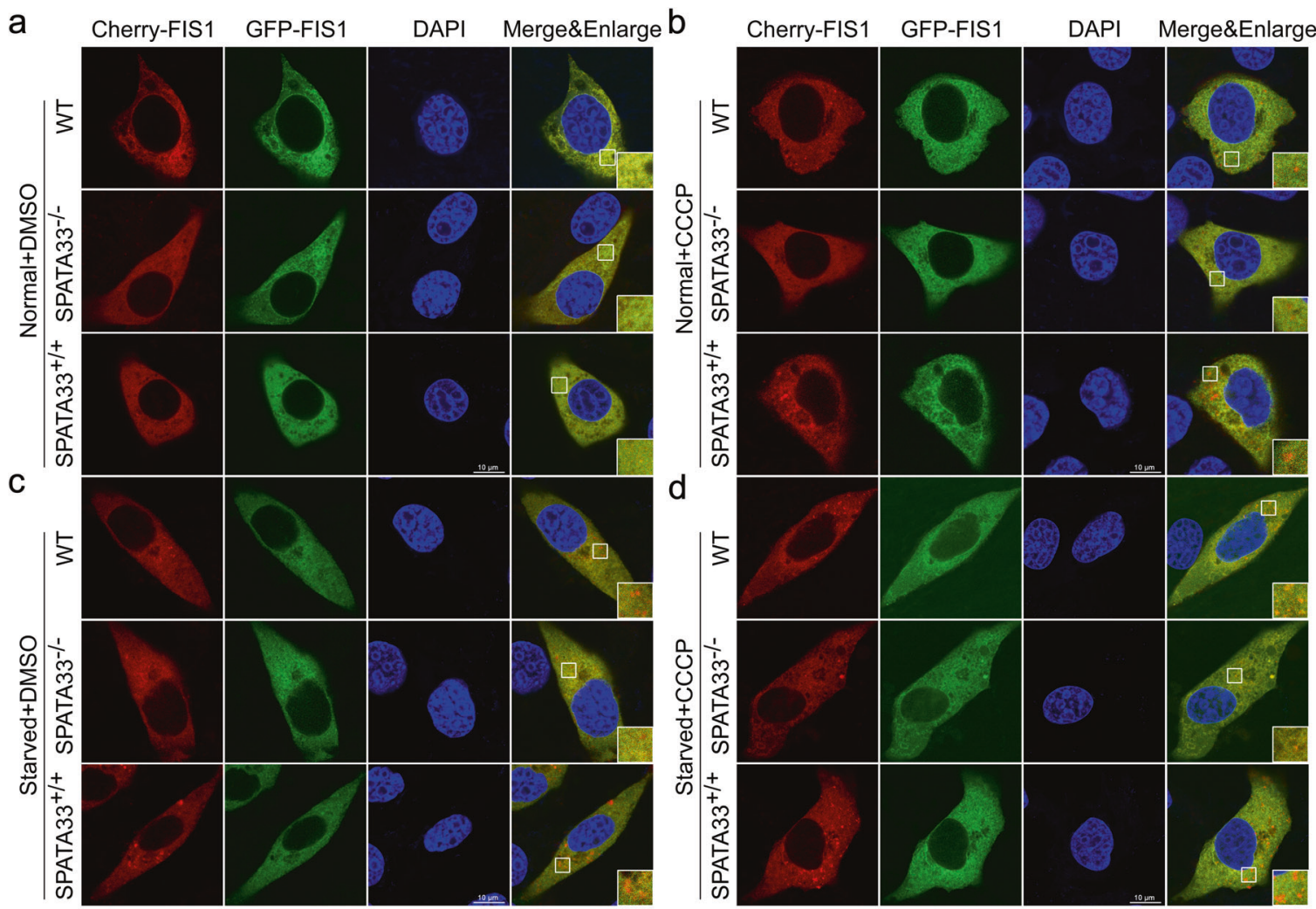

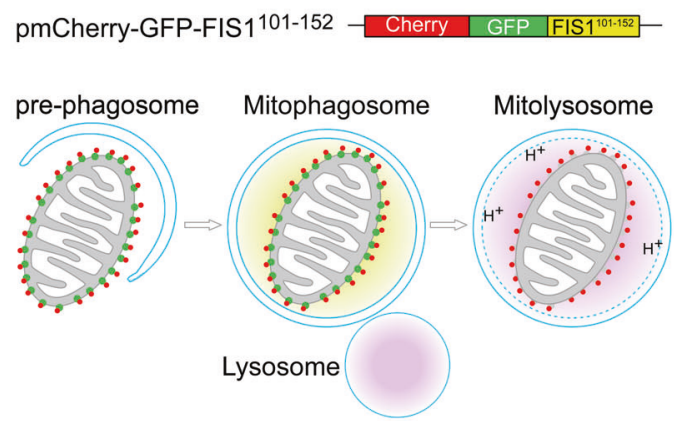

Fig. 5 MitoQC-based analysis. a-d Fluorescent microscopy of the pmCherry-GFP-FIS1 $1^{101-152}$ tandem reporter in Spata33 knockout $\left(\right.$ spata33 $^{-/-}$, \#33), Spata33 overexpression (spata33 $3^{+/+}$), and wildtype GC-1 cell lines. The cells were transfected with the pmCherryGFP-FIS $1^{101-152}$ and cultured in normal (control), CCCP $(10 \mu \mathrm{M}, 1 \mathrm{~h})$, EBSS medium $(1 \mathrm{~h})$ or EBSS with CCCP $(10 \mu \mathrm{M}, 1 \mathrm{~h})$ addition, respectively. Single channel (red, green, or blue) and merged images were taken by confocal microscopy. The insets showed an enlarged view of the indicated squares. Scale bar: $10 \mu \mathrm{m}$. e Schematic diagram of MitoQC-based analysis principle. pmCherry-GFP-FIS1 $1^{101-152}$

The SPATA33-mediated mitophagy is male germline specific, which could play important roles in spermatogenesis. The autophagy mediator SPATA33 is specifically expressed in spermatogenic cells, including spermatogonia, spermatocytes, round spermatids, and spermatozoa during spermatogenesis, in which key autophagy machinery ATG16L1 and $\mathrm{LC} 3 \mathrm{~B}$, and mitochondrial outer membrane protein

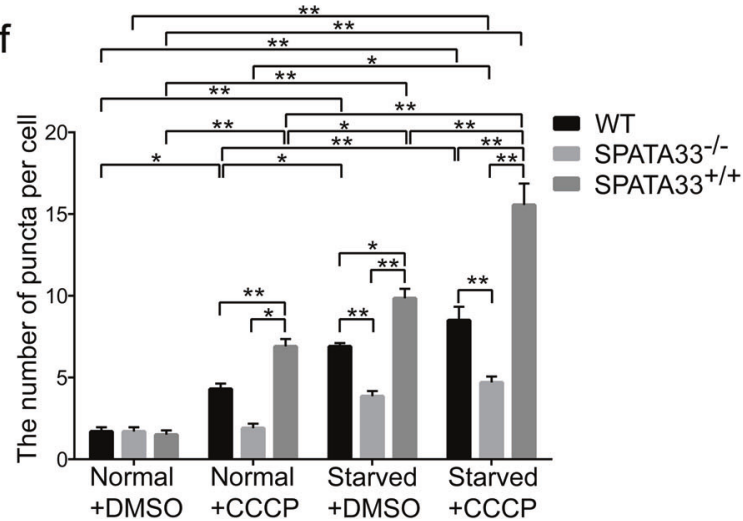

tandem reporter expresses a tandem mCherry-GFP tag fused to the mitochondrial outer membrane protein FIS1, MitoQC displays red and green fluorescence during steady-state conditions, but the mCherry signals become stable when mitophagy is induced, because mitochondria are delivered to the lysosome where the GFP signals are quenched. Therefore, mCherry-only puncta are seen during mitophagy activation. $\mathbf{f}$ Statistical analysis of vesicles positive for mCherry puncta (mitolysosome) ( $>15$ cells per experiment) by $t$-test in the $(\mathbf{a}-\mathbf{d})$. The mean $\pm \mathrm{SD}$ are from three independent experiments. $* p<0.05 ;{ }^{*} p<$ 0.01 .

VDAC2 are also expressed. In addition, SP1 specifically binds to the Spata33 promoter region in vivo and activates its expression during spermatogenesis. Notably, SPATA33 promotes mitophagy as well as being an autophagy mediator. These results support its role in mitophagy in the germline development and differentiation. As autophagy is indispensable for spermatogenesis [58], identification of the 

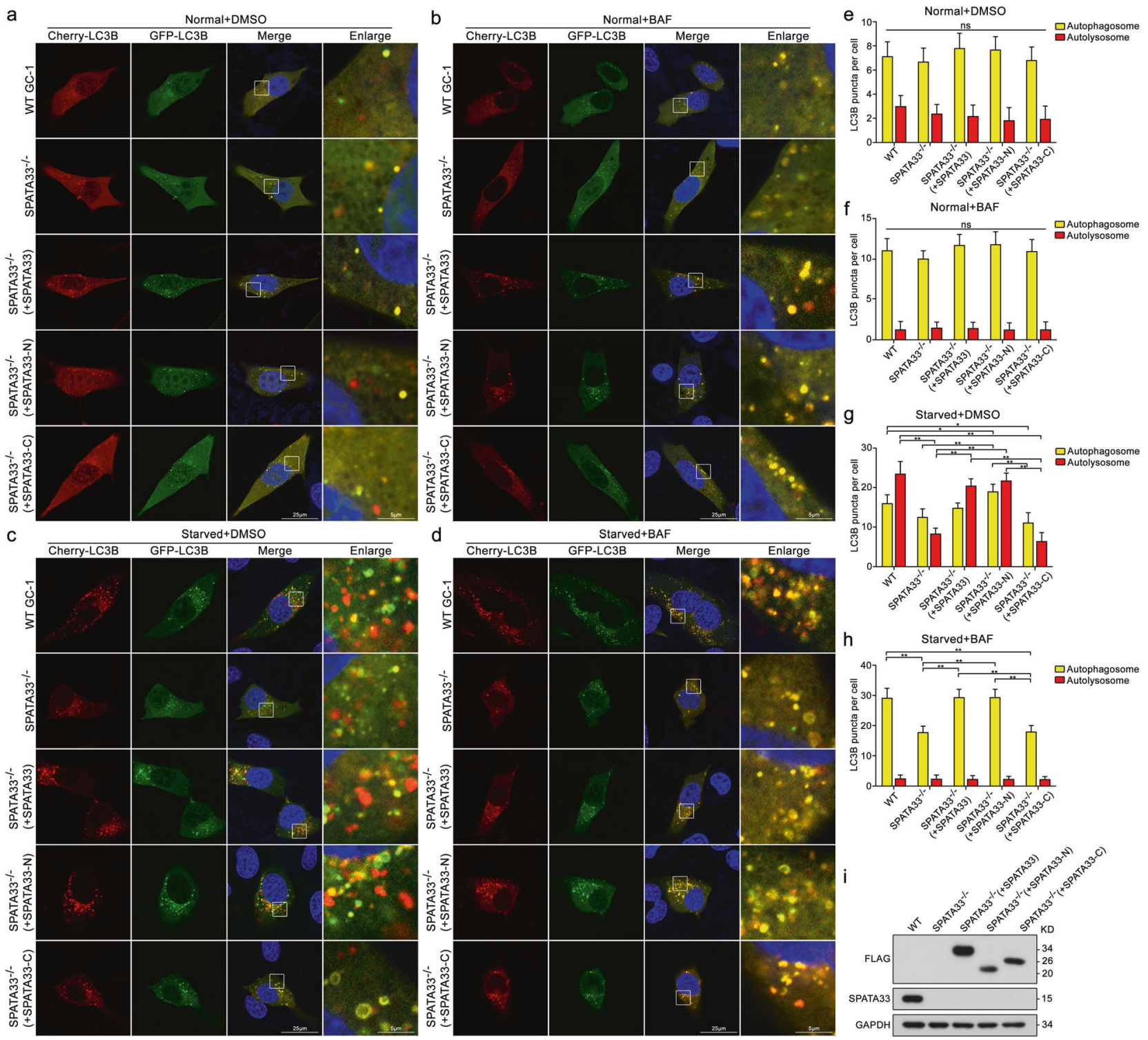

Fig. 6 SPATA33-associated autophagy flux. a-d The pmCherryGFP-LC3B tandem reporter analysis in Spata33 knockout

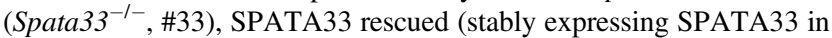
Spata33 $^{-l-}$ cell lines), SPATA33-N rescued (stably expressing SPATA33-N in Spata33 $^{-1-}$ cell lines), SPATA33-C rescued (stably expressing SPATA33-C in Spata33 ${ }^{-1-}$ cell lines), and wild-type GC-1 cell lines. Representative images of the cells transfected with the pmCherry-GFP-LC3B reporter and cultured in normal (control), bafilomycin A1 (100 $\mathrm{nM}$ ) addition (1 h), EBSS medium (1 h), or EBSS with bafilomycin A1 $(100 \mathrm{nM})$ addition $(1 \mathrm{~h})$, respectively. Single

autophagy mediator SPATA33 in germline provides a new understanding of maintaining cellular homeostasis during spermatogenesis through mitophagy. In addition, paternal mitochondria removal in zygote is a key step to ensure maternal inheritance of mitochondria. Both ubiquitindependent [47, 48] and ubiquitin-independent mitophagy [51] have been observed in the removal process. As the SPATA33-mediated mitophagy is ubiquitin-independent, channel (red, green or blue) and merged images were taken by confocal microscopy. Scale bar: $25 \mu \mathrm{m}$, scale bar in enlarged panels: $5 \mu \mathrm{m}$. e-h Statistical analysis of vesicles positive for both GFP and mCherry (autophagosomes) and for mCherry (autolysosomes) ( $>15$ cells per experiment) by $t$-test in the (a-d), respectively. The mean \pm SD are from three independent experiments. ${ }^{*} p<0.05 ; * * p<0.01$. i Determination of effectively rescuing of SPATA33-N and SPATA33-C. Endogenous SPATA33, FLAG-SPATA33, FLAG-SPATA33-N, and FLAG-SPATA33-C protein levels were analyzed by western blotting.

whether SPATA33-mediated mitophagy is involved in paternal mitochondria removal in mammals remains an open question.

In summary, we identify a new kind of autophagy mediator SPATA33 in germline, which promotes mitophagy as well. The SPATA33-mediated mitophagy broadens our understanding of selective autophagy and mitochondrial homeostasis. 


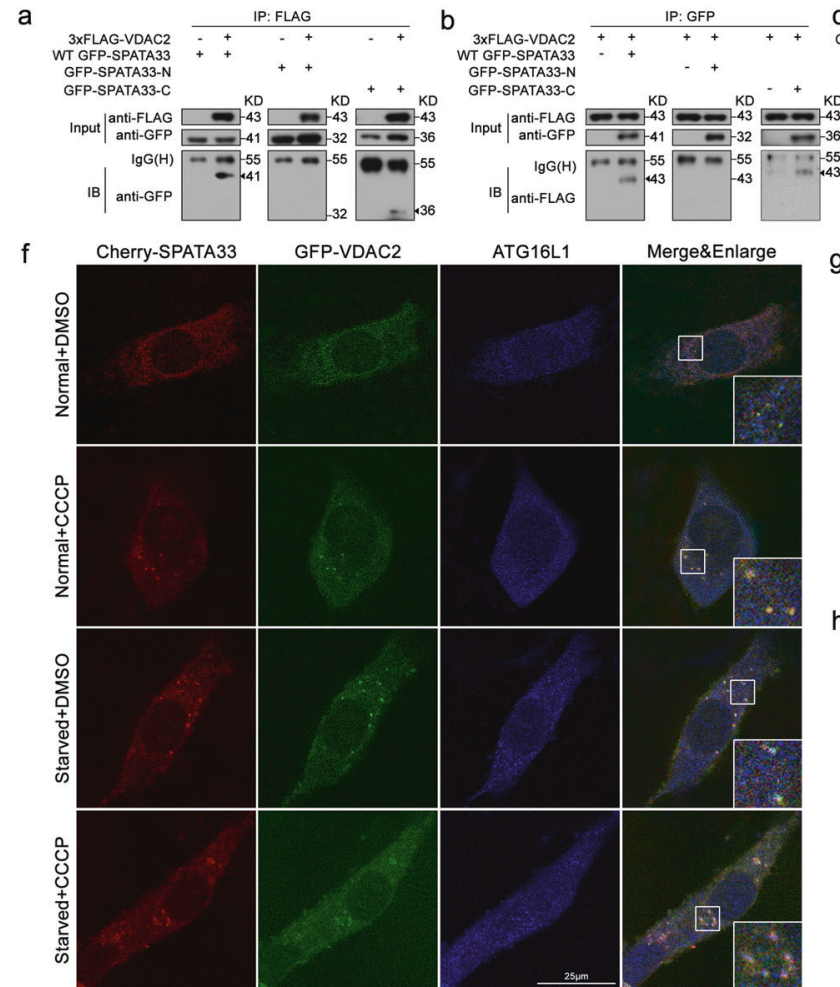

Fig. 7 SPATA33 interaction with ATG16L1 and VDAC2. a, b Coimmunoprecipitation between VDAC2 and deletion mutants of SPATA33. pCherry-FLAG-ATG16L1 was transiently co-transfected with pGFP-SPATA33, pGFP-N-SPATA33, or pGFP-C-SPATA33 in HEK293T cells, respectively. Cell lysates were examined by western blotting using the anti-FLAG or anti-GFP antibody. For coimmunoprecipitation, the lysates were immunoprecipitated with anti-FLAG or anti-GFP, followed by immunoblotting with the anti-GFP or antiFLAG antibody. Arrowheads indicate the target bands. c, d Coimmunoprecipitation between ATG16L1 and deletion mutants of SPATA33. p3xFLAG-VDAC2 was transiently co-transfected with pGFP-SPATA33, pGFP-N-SPATA33, or pGFP-C-SPATA33 in HEK293T cells, respectively. Cell lysates were examined by western blotting using the anti-FLAG or anti-GFP antibody. For coimmunoprecipitation, the lysates were immunoprecipitated with anti-FLAG or anti-GFP, followed by immunoblotting with the anti-GFP or antiFLAG antibody. Arrowheads indicate the target bands. e Coimmunoprecipitation analysis of interaction among endogenous SPATA33, ATG16L1, and VDAC2 in GC-1 cells. The GC-1 cell lysates were immunoprecipitated with anti-SPATA33, anti-ATG16L1, or antiVDAC2 antibody, followed by immunoblotting with the antiSPATA33, anti-VDAC2, or anti-ATG16L1 antibody, respectively.

\section{Materials and methods}

\section{Animals}

Mouse strain ICR was purchased from the Wuhan Disease Prevention Center. The strain Kunming White was purchased from the Animal Center of the Wuhan Zhongnan Hospital. All animals were raised in the animal center with P3 grade in Wuhan University. The research is conducted in accordance with the guiding principles for biomedical research involving animals of Ethics and Animal Welfare

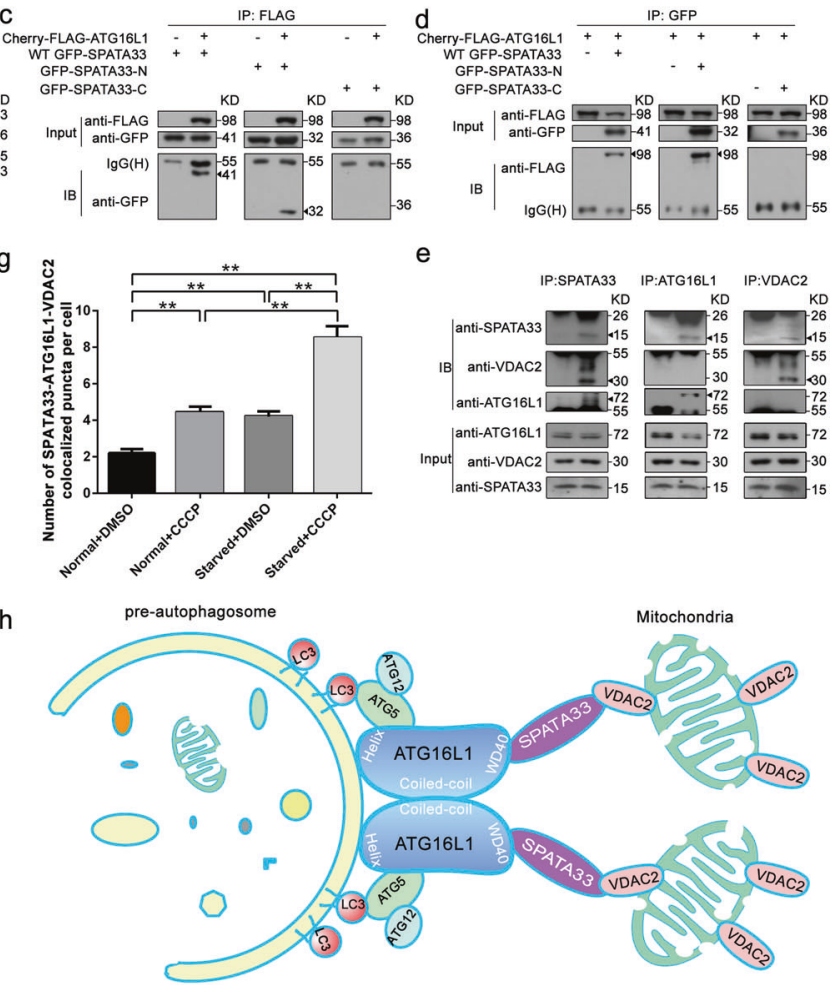

The whole cell lysates were examined by western blotting using the anti-ATG16L1, anti-VDAC2, or anti-SPATA33 antibody. Arrowheads indicate the target bands. $\mathbf{f}$ Colocalization analysis of SPATA33 with VDAC2 and ATG16L1. GC-1 cells were transiently co-transfected with pCherry-SPATA33 and pGFP-VDAC2. After $24 \mathrm{~h}$ in normal medium, the cells were cultured in normal (control), CCCP $(10 \mu \mathrm{M}, 1$ h), EBSS medium $(1 \mathrm{~h})$, or EBSS with CCCP $(10 \mu \mathrm{M}, 1 \mathrm{~h})$ addition, respectively. Immunofluorescence analysis was performed with antiATG16L1 and Dylight 405 Donkey anti-Rabbit IgG $(\mathrm{H}+\mathrm{L})$ antibodies. Single channel (red, green, or blue) and merged images were taken by confocal microscopy. Colocalizing structures are indicated in white (merge). Scale bar: $25 \mu \mathrm{m}$. g Statistical analysis of colocalized puncta between SPATA33, ATG16L1, and VDAC2. Data are presented as means \pm S.D. ${ }^{*} p<0.01 \quad(n=3$ independent experiments, $>15$ cells per experiment). h SPATA33 mediates mitophagy via interaction with VDAC2 and ATG16L1. SPATA33 interacts with mitochondrial outer membrane protein VDAC2 through its carboxyl terminal, while its amino terminal interacts with WD40 region of ATG16L1 protein. Upon starvation stress, damaged mitochondria are encapsulated by autophagosome through mediator SPATA33 linking between VDAC2 and ATG16L1 to initiate mitophagy.

Committee of College of Life Sciences of Wuhan University and the committee.

\section{Antibodies and reagents}

Primary antibodies: Anti-SPATA33 was prepared by Wuhan Virus Research Institute of CAS, Wuhan, China. Anti-LC3B (3868, Cell Signaling Technology, Danvers, USA), Anti-ATG16L1 (8089, Cell Signaling Technology), Anti-GAPDH (CW0100, CWBIO, Beijing, China), AntiFLAG (F3165, Sigma-Aldrich, St Louis, USA), Anti-MYC 
(11667149001, Roche Applied Science, Indianapolis, USA), Anti-GFP (11814460001, Roche Applied Science), Anti-VDAC2 (11663-1-AP, Proteintech Group, Rosemont, USA), Anti-SP1 (ab13370, Abcam Inc., Cambridge, USA), Rabbit Polyclonal ATG16L1 Antibody [Alexa Fluor ${ }^{\circledR} 488$ ] (NB110-60928AF488, NOVUS, Beijing, China), Rabbit Polyclonal LC3B Antibody [Alexa Fluor 488] (NB1002220AF488, NOVUS, Beijing, China), COX-IV Monoclonal Antibody (YM0162, ImmunoWay, USA), AntiSQSTM1 (A19700, Abclonal, Wuhan, China).

Secondary antibodies: Peroxidase-conjugated AffiniPure goat anti-mouse IgG, light chain specific (115-035174, Jackson ImmunoResearch Laboratories, West Grove, USA), Peroxidase-conjugated AffiniPure fragment rabbit anti-mouse $\operatorname{IgG}$, Fc fragment specific (315-036-046, Jackson ImmunoResearch Laboratories), AMCAconjugated AffiniPure goat anti-mouse $\operatorname{IgG}(\mathrm{H}+\mathrm{L})$ (31430, Pierce Biotechnology Company, Carlsbad, USA), Goat anti-rabbit IgG horseradish peroxidase (HRP)-linked whole antibody (31460, Pierce Biotechnology Company). Fluorescein antibodies: FITC-conjugated ImmunoPure goat anti-rabbit IgG $(\mathrm{H}+\mathrm{L})$ (ZF-0311, Zhongshan, Beijing, China), TRITC-conjugated ImmunoPure goat anti-rabbit $\operatorname{IgG}(\mathrm{H}+\mathrm{L})$ (ZF-0316, Zhongshan), and Dylight 405 Donkey anti-rabbit IgG $(\mathrm{H}+\mathrm{L})$ (ANT081, Antgene, Wuhan, China).

Reagents: MitoTracker Red CMXRos (167095-09-2, YESEN, Shanghai, China), CCCP (T7081, TargetMol, Shanghai, China), and Bafilomycin A1 (B1793, SigmaAldrich, USA).

\section{Plasmid constructs and gRNA design}

Full-length SPATA33 (NM_177279.4) was cloned into pCMV-Tag2B-3xFLAG, pcDNA3.0-MYC, and pSicoCherry-FLAG using EcoRI and XhoI to generate p3x FLAG-SPATA33, pMYC-SPATA33, and pCherry-FLAGSPATA33, respectively, while pSico-GFP-SPATA33 was made by XhoI and EcoRI digestions. Full-length SPATA33, N-60aa-SPATA33, and C-73aa-SPATA33 were cloned into pEGFP-N1 using XhoI and EcoRI to generate pGFPSPATA33, pGFP-SPATA33-N, and pGFP-SPATA33-C. Full-length SPATA33, N-60aa-SPATA33, and C-73aaSPATA33 were cloned into plove-CMV using XhoI and EcoRI to generate plove-SPATA33, plove-SPATA33-N, and plove-SPATA33-C. Full-length ATG16L1 (NM_ 001205391.1), N-306aa-ATG16L1, and C-317aa-ATG16L1 were digested with SalI and HindIII, while the pSicoCherry-FLAG and pEGFP-N1 were digested with XhoI and HindIII, and were cloned to generate pCherry-FLAGATG16L1, pGFP-ATG16L1-N, and pGFP-ATG16L1-C, respectively. 101st to 152 nd amino acids in Fis 1
(NM_025562.3) were cloned into pmCherry-GFP-LC3B-m (P0202, MiaoLing Plasmid Sharing Platform, Wuhan, China) using HindIII and $M f e I$ to generate pmCherry-GFPFIS $1^{101-152}$.

LentiCRISPRv2-SPATA33-gRNA and LentiCRISPRv2ATG16L1-gRNA were constructed as described previously [59, 60]. Briefly, SPATA33-gRNAs and ATG16L1-gRNAs were designed according to CRISPR Design Tool (http:// crispr.mit.edu/) and synthesized with BsmBI sticky end, then annealed and inserted into the lentiCRISPRv2 plasmid digested with BsmBI (Fermentas, Vilnius, Lithuania). p3xFLAG-VDAC2 and pGFP-VDAC2 constructs were constructed as our previous study [61]. All the primers are described in Table S1.

\section{Cell culture and transfection}

HEK293T, TM4, GC-1, and HeLa cells obtained from China Center for Type Culture Collection. The cells were cultured in high glucose DMEM (SH30022.01B, HyClone, Logan, USA) with 12\% FBS (P30-330250, PAN-Biotech, Aidenbach, Germany) at $37^{\circ} \mathrm{C}$ in a $5 \%$ $\mathrm{CO}_{2}$ in cell incubator. For transfection, cells were cultured in cell plates or glass cover slides. Lipofectamine 2000 (11668027, Invitrogen) was used in each well. For starvation treatments, the cells were cultured in EBSS (Cat\# SH30029.02, HyClone, Logan, USA) for various times.

\section{Western blot analysis and coimmunoprecipitation assays}

Western blot analysis was performed using routine protocols. Protein extracts from cells were separated in $12 \%$ SDS-polyacrylamide gels and then transferred onto 0.45 $\mu \mathrm{m}$ membranes (NK0414, Roche Diagnostics, Indianapolis, IN, USA). Primary antibodies were incubated with the membranes overnight at $4{ }^{\circ} \mathrm{C}$. The membranes were washed in TBST ( $20 \mathrm{mM}$ Tris- $\mathrm{HCl} \mathrm{pH} 7.5,150 \mathrm{mM} \mathrm{NaCl}$, $0.1 \%$ Tween 20) three times, incubated with the indicated HRP-conjugated secondary antibody for $1 \mathrm{~h}$ at room temperature, and then washed in TBST five times. A Super Signal Chemiluminescent Substrate system (K-12045D50, Advansta, Menlo Park, USA) was used to detect the signals.

Coimmunoprecipitation was used to analyze protein interactions in vitro. HEK293T cells were co-transfected with related plasmid DNAs. After $48 \mathrm{~h}$, the cells were lysed in IP buffer $(50 \mathrm{mM}$ Tris- $\mathrm{HCl}$ at $\mathrm{pH} 8.0,0.15 \mathrm{M} \mathrm{NaCl}, 1$ mM EGTA, and $0.5 \%$ NP-40) containing protease inhibitor cocktail (04693159001, Roche Applied Science, Indianapolis, USA). The other steps are described in the previous study [62]. 


\section{Immunofluorescence analysis}

Testis tissues were embedded in OCT medium (4583, Tissue-Tek, Miles, USA) and cut into a series of $8 \mu \mathrm{m}$ sections using a cryostat (Leica, Bensheim, Germany). GC-1, TM4 and HeLa cells were cultured on glass cover slides. Both sections and cover slides were fixed with 4\% PFA for 20 min at room temperature, then permeabilized with $1 \%$ Triton X-100 (0.1\% Triton for cell lines) (9002-93-1, Sigma-Aldrich) in PBS for $30 \mathrm{~min}$. The other steps were described in the previous study [56]. Images were taken by confocal fluorescence microscopy (SP8, Leica).

\section{MitoQC-based assays}

GC-1 cells (wild type, Spata33-overexpressed, and Spata33 KO cell lines) were cultured on cover slides and transfected with the pmCherry-GFP-FIS1 ${ }^{101-152}$. After $24 \mathrm{~h}$ in normal culture, the cells were treated with normal medium (control), CCCP (10 $\mu \mathrm{M}, 1 \mathrm{~h})$, EBSS medium (1 h), and EBSS with CCCP $(10 \mu \mathrm{M}, 1 \mathrm{~h})$ addition, respectively. The other steps were described in the immunofluorescence analysis. Images were taken by confocal fluorescence microscopy (SP8, Leica).

\section{Flow cytometry}

Testicular tissue was removed under aseptic conditions from adult male mice and placed in a petri dish containing precooled PBS. Testicular white membrane was removed with pointed tweezers under sterile conditions, and the samples were shred in another clean petri dish within $0.25 \%$ trypsin and $0.2 \%$ collagenase I, digested with shaking in 37 ${ }^{\circ} \mathrm{C}$ incubator for $10-20 \mathrm{~min}$. After that, the digestion was stopped by adding $4 \mathrm{ml}$ DMEM/F12 medium and 20\% FBS medium. The sample were blown and beaten repeatedly until the cells dispersed, and passed through a $70 \mu \mathrm{m}$ filter into a new centrifuge tube, then centrifuged at $1800 \mathrm{rpm}$ for $10 \mathrm{~min}$ at room temperature. After supernatant discarded, precipitation was resuspended in $4 \mathrm{ml}$ medium with $20 \%$ FBS and penicillin-streptomycin. Flow cytometry (SH800S, SONY, Japan) was used for sorting based on the physical properties of the cells (the size and granule density of the cells).

\section{Semi-quantitative RT-PCR}

TRIzol (15596-026, Invitrogen) was used to isolate total RNA, which was transcribed using a poly (T)18 primer and MMLV (M1701, Promega). Specific primers were designed to amplify the Spata33 gene by PCR instrument $\left(\mathrm{S}_{1000}{ }^{\mathrm{TM}}\right.$ Thermal Cycler, BIO-RAD, California, USA). Actin was amplified as a control. The primer sequences are described in Table S1.

\section{Lentivirus generation, infection, and gene knockout}

To generate lentivirus as described before, the HEK293T cells seeded on 100-mm plate were transfected with lentiCRISPRv2-SPATA33-gRNA and lentiviral packaging vectors pRSV-Rev (12253, Addgene, Watertown, USA), pMD2.G (12259, Addgene), and pCMV-VSV-G (8454, Addgene) using Lipofectamine 2000 according to the manufacturer's instructions. After incubation for $48 \mathrm{~h}$, the supernatants were filtered through $0.45 \mu \mathrm{m}$ filters and used directly to infect TM4 or GC-1 cells. Puromycin (A1113802, Fisher Scientific, Waltham, USA) was used to screen the cells. The primer sequences are described in Table S1.

\section{Transmission electron microscope}

After treated with starvation and CCCP $(10 \mu \mathrm{M})$ for $1 \mathrm{~h}$, GC-1 cells (WT and Spata33-overexpressed) were fixed with $3 \%$ PFA, $1.5 \%$ glutaraldehyde, and $2.5 \%$ sucrose, then washed with PB buffer (19 ml: $0.2 \mathrm{M} \mathrm{NaH}_{2} \mathrm{PO}_{4} ; 81 \mathrm{ml}: 0.2$ $\mathrm{M} \mathrm{Na}_{2} \mathrm{HPO}_{4}$ ) for 3 times, each time for $5 \mathrm{~min}$. The cells were collected by gradient centrifugation at $4{ }^{\circ} \mathrm{C}$, and immobilized with osmic acid in ice bath for $1 \mathrm{~h}$. The cells were stained overnight with uranium dioxy acetate, and gradient dehydration was followed. The resin was embedded and hardened, then the samples were sectioned at a thickness of $70 \mathrm{~nm}$ using an ultramicrotome (EM UC7, Leica) and observed by transmission electron microscope (Tecnai $\mathrm{G}^{2}$ 20, FEI, Oregon, USA).

Funding This work was supported by the National Key R\&D Program of China (2019YFA0802500) and the National Natural Science Foundation of China (31771487, 31771370, and 31970539).

Author contributions Conceptualization, $\mathrm{HC}$ and RZ; methodology, $\mathrm{YZ}$ and $\mathrm{XX}$; formal analysis, $\mathrm{YZ}, \mathrm{XX}$, and RZ; investigation, $\mathrm{YZ}, \mathrm{XX}$, $\mathrm{MH}$, and $\mathrm{XW}$; writing - original draft, $\mathrm{YZ}$; writing-review \& editing, $\mathrm{YZ}$ and RZ; supervision and funding, $\mathrm{HC}$ and RZ; project administration, $\mathrm{HC}$ and $\mathrm{RZ}$.

\section{Compliance with ethical standards}

Conflict of interest The authors declare that they have no conflict of interest.

Ethical approval All animal experiments and methods were performed in accordance with the relevant approved guidelines and regulations, as well as under the approval of the Ethics Committee of Wuhan University.

Publisher's note Springer Nature remains neutral with regard to jurisdictional claims in published maps and institutional affiliations. 
Open Access This article is licensed under a Creative Commons Attribution 4.0 International License, which permits use, sharing, adaptation, distribution and reproduction in any medium or format, as long as you give appropriate credit to the original author(s) and the source, provide a link to the Creative Commons license, and indicate if changes were made. The images or other third party material in this article are included in the article's Creative Commons license, unless indicated otherwise in a credit line to the material. If material is not included in the article's Creative Commons license and your intended use is not permitted by statutory regulation or exceeds the permitted use, you will need to obtain permission directly from the copyright holder. To view a copy of this license, visit http://creativecommons. org/licenses/by/4.0/.

\section{References}

1. Hermo L, Pelletier RM, Cyr DG, Smith CE. Surfing the wave, cycle, life history, and genes/proteins expressed by testicular germ cells. Part 1: background to spermatogenesis, spermatogonia, and spermatocytes. Microsc Res Tech. 2010;73:241-78.

2. Gao H, Khawar MB, Li W. Essential role of autophagy in resource allocation during sexual reproduction. Autophagy. 2020;16:18-27.

3. Da Ros M, Lehtiniemi T, Olotu O, Fischer D, Zhang FP, Vihinen H, et al. FYCO1 and autophagy control the integrity of the haploid male germ cell-specific RNP granules. Autophagy. 2017;13:302-21.

4. Youle RJ, Narendra DP. Mechanisms of mitophagy. Nat Rev Mol Cell Biol. 2011;12:9-14.

5. Liu J, Liu W, Li R, Yang H. Mitophagy in parkinson's disease: from pathogenesis to treatment. Cells. 2019;8:712.

6. Lou G, Palikaras K, Lautrup S, Scheibye-Knudsen M, Tavernarakis N, Fang EF. Mitophagy and neuroprotection. Trends Mol Med. 2020;26:8-20.

7. Jiang S, Sun J, Mohammadtursun N, Hu Z, Li Q, Zhao Z, et al. Dual role of autophagy/mitophagy in chronic obstructive pulmonary disease. Pulm Pharmacol Ther. 2019;56:116-25.

8. Yang M, Linn BS, Zhang Y, Ren J. Mitophagy and mitochondrial integrity in cardiac ischemia-reperfusion injury. Biochim Biophys Acta Mol Basis Dis. 2019;1865:2293-302.

9. Wei PZ, Szeto CC. Mitochondrial dysfunction in diabetic kidney disease. Clin Chim Acta. 2019;496:108-16.

10. Liu ML, Wang JL, Wei J, Xu LL, Yu M, Liu XM, et al. Tri-orthocresyl phosphate induces autophagy of rat spermatogonial stem cells. Reproduction. 2015;149:163-70.

11. Xu LL, Liu ML, Wang JL, Yu M, Chen JX. Saligenin cyclic-otolyl phosphate (SCOTP) induces autophagy of rat spermatogonial stem cells. Reprod Toxicol. 2016;60:62-8.

12. Mancilla H, Maldonado R, Cereceda K, Villarroel-Espindola F, Montes de Oca M, Angulo C, et al. Glutathione depletion induces spermatogonial cell autophagy. J Cell Biochem. 2015;116:2283-92.

13. Liu K, Zhang G, Wang Z, Liu Y, Dong J, Dong X, et al. The protective effect of autophagy on mouse spermatocyte derived cells exposure to $1800 \mathrm{MHz}$ radiofrequency electromagnetic radiation. Toxicol Lett. 2014;228:216-24.

14. Zhang G, Ling X, Liu K, Wang Z, Zou P, Gao J, et al. The peIF2alpha/ATF4 pathway links endoplasmic reticulum stress to autophagy following the production of reactive oxygen species in mouse spermatocyte-derived cells exposed to dibutyl phthalate. Free Radic Res. 2016;50:698-707.

15. Aparicio IM, Munoz PM, Salido GM, Pena FJ, Tapia JA. The autophagy-related protein LC3 is processed in stallion spermatozoa during short-and long-term storage and the related stressful conditions. Animal. 2016;10:1182-91.
16. Bolanos JM, Moran AM, da Silva CM, Davila MP, Munoz PM, Aparicio IM, et al. During cooled storage the extender influences processed autophagy marker light chain 3 (LC3B) of stallion spermatozoa. Anim Reprod Sci. 2014;145:40-6.

17. Guo X, Shen J, Xia Z, Zhang R, Zhang P, Zhao C, et al. Proteomic analysis of proteins involved in spermiogenesis in mouse. J Proteome Res. 2010;9:1246-56.

18. Guo X, Zhang P, Qi Y, Chen W, Chen X, Zhou Z, et al. Proteomic analysis of male $4 \mathrm{C}$ germ cell proteins involved in mouse meiosis. Proteomics. 2011;11:298-308.

19. Gao F, Li G, Liu C, Gao H, Wang H, Liu W, et al. Autophagy regulates testosterone synthesis by facilitating cholesterol uptake in Leydig cells. J Cell Biol. 2018;217:2103-19.

20. Wang H, Wan H, Li X, Liu W, Chen Q, Wang Y, et al. Atg7 is required for acrosome biogenesis during spermatogenesis in mice. Cell Res. 2014;24:852-69.

21. Sidjanin DJ, Park AK, Ronchetti A, Martins J, Jackson WT. TBC1D20 mediates autophagy as a key regulator of autophagosome maturation. Autophagy. 2016;12:1759-75.

22. Ferder IC, Fung L, Ohguchi Y, Zhang X, Lassen KG, Capen D, et al. Meiotic gatekeeper STRA8 suppresses autophagy by repressing Nr1d1 expression during spermatogenesis in mice. PLoS Genet. 2019;15:e1008084.

23. Liu C, Wang H, Shang Y, Liu W, Song Z, Zhao H, et al. Autophagy is required for ectoplasmic specialization assembly in Sertoli cells. Autophagy. 2016;12:814-32.

24. Shang Y, Wang H, Jia P, Zhao H, Liu C, Liu W, et al. Autophagy regulates spermatid differentiation via degradation of PDLIM1. Autophagy. 2016;12:1575-92.

25. Kitada T, Asakawa S, Hattori N, Matsumine H, Yamamura Y, Minoshima $\mathrm{S}$, et al. Mutations in the parkin gene cause autosomal recessive juvenile parkinsonism. Nature. 1998;392:605-8.

26. Durcan TM, Fon EA. USP8 and PARK2/parkin-mediated mitophagy. Autophagy. 2015;11:428-9.

27. Wong YC, Holzbaur EL. Optineurin is an autophagy receptor for damaged mitochondria in parkin-mediated mitophagy that is disrupted by an ALS-linked mutation. Proc Natl Acad Sci USA. 2014;111:E4439-48.

28. Lazarou M, Sliter DA, Kane LA, Sarraf SA, Wang C, Burman JL, et al. The ubiquitin kinase PINK1 recruits autophagy receptors to induce mitophagy. Nature. 2015;524:309-14.

29. Araya J, Tsubouchi K, Sato N, Ito S, Minagawa S, Hara H, et al. PRKN-regulated mitophagy and cellular senescence during COPD pathogenesis. Autophagy. 2019;15:510-26.

30. Li G, Yang J, Yang C, Zhu M, Jin Y, McNutt MA, et al. PTENalpha regulates mitophagy and maintains mitochondrial quality control. Autophagy. 2018;14:1742-60.

31. Wang Y, Serricchio M, Jauregui M, Shanbhag R, Stoltz T, Di Paolo CT, et al. Deubiquitinating enzymes regulate PARK2mediated mitophagy. Autophagy. 2015;11:595-606.

32. Chen Z, Liu L, Cheng Q, Li Y, Wu H, Zhang W, et al. Mitochondrial E3 ligase MARCH5 regulates FUNDC1 to fine-tune hypoxic mitophagy. EMBO Rep. 2017;18:495-509.

33. Wu H, Wang Y, Li W, Chen H, Du L, Liu D, et al. Deficiency of mitophagy receptor FUNDC1 impairs mitochondrial quality and aggravates dietary-induced obesity and metabolic syndrome. Autophagy. 2019;15:1882-98.

34. Kanki T, Wang K, Cao Y, Baba M, Klionsky DJ. Atg32 is a mitochondrial protein that confers selectivity during mitophagy. Dev Cell. 2009;17:98-109.

35. Okamoto K, Kondo-Okamoto N, Ohsumi Y. Mitochondriaanchored receptor Atg32 mediates degradation of mitochondria via selective autophagy. Dev Cell. 2009;17:87-97.

36. Gatica D, Lahiri V, Klionsky DJ. Cargo recognition and degradation by selective autophagy. Nat Cell Biol. 2018;20:233-42. 
37. Murakawa T, Yamaguchi O, Hashimoto A, Hikoso S, Takeda T, Oka $\mathrm{T}$, et al. Bcl-2-like protein 13 is a mammalian Atg32 homologue that mediates mitophagy and mitochondrial fragmentation. Nat Commun. 2015;6:7527.

38. Hanna RA, Quinsay MN, Orogo AM, Giang K, Rikka S, Gustafsson AB. Microtubule-associated protein 1 light chain 3 (LC3) interacts with Bnip3 protein to selectively remove endoplasmic reticulum and mitochondria via autophagy. J Biol Chem. 2012;287:19094-104.

39. Schweers RL, Zhang J, Randall MS, Loyd MR, Li W, Dorsey FC, et al. NIX is required for programmed mitochondrial clearance during reticulocyte maturation. Proc Natl Acad Sci USA. 2007;104:19500-5.

40. Novak I, Kirkin V, McEwan DG, Zhang J, Wild P, Rozenknop A, et al. Nix is a selective autophagy receptor for mitochondrial clearance. EMBO Rep. 2010;11:45-51.

41. Bhujabal Z, Birgisdottir AB, Sjottem E, Brenne HB, Overvatn A, Habisov S, et al. FKBP8 recruits LC3A to mediate Parkinindependent mitophagy. EMBO Rep. 2017;18:947-61.

42. Liu L, Feng D, Chen G, Chen M, Zheng Q, Song P, et al. Mitochondrial outer-membrane protein FUNDC1 mediates hypoxia-induced mitophagy in mammalian cells. Nat Cell Biol. 2012;14:177-85.

43. Lahiri V, Klionsky DJ. PHB2/prohibitin 2: an inner membrane mitophagy receptor. Cell Res. 2017;27:311-2.

44. Wei Y, Chiang WC, Sumpter R Jr., Mishra P, Levine B. Prohibitin 2 is an inner mitochondrial membrane mitophagy receptor. Cell. 2017;168:224-38.10

45. Lu H, Li G, Liu L, Feng L, Wang X, Jin H. Regulation and function of mitophagy in development and cancer. Autophagy. 2013;9:1720-36.

46. Sutovsky P, Song WH. Post-fertilisation sperm mitophagy: the tale of mitochondrial Eve and Steve. Reprod Fertil Dev. 2017;30:56-63

47. Song WH, Yi YJ, Sutovsky M, Meyers S, Sutovsky P. The ART and science of sperm mitophagy. Autophagy.2016;12:2510-1.

48. Song WH, Yi YJ, Sutovsky M, Meyers S, Sutovsky P. Autophagy and ubiquitin-proteasome system contribute to sperm mitophagy after mammalian fertilization. Proc Natl Acad Sci USA. 2016;113: E5261-70.

49. Politi Y, Gal L, Kalifa Y, Ravid L, Elazar Z, Arama E. Paternal mitochondrial destruction after fertilization is mediated by a common endocytic and autophagic pathway in Drosophila. Dev Cell. 2014;29:305-20.

50. Djeddi A, Al Rawi S, Deuve JL, Perrois C, Liu YY, Russeau M, et al. Sperm-inherited organelle clearance in C. elegans relies on LC3-dependent autophagosome targeting to the pericentrosomal area. Development. 2015;142:1705-16.

51. Lim Y, Rubio-Pena K, Sobraske PJ, Molina PA, Brookes PS, Galy V, et al. Fndc-1 contributes to paternal mitochondria elimination in C. elegans. Dev Biol. 2019;454:15-20.

52. Chen H, Yi M, Sheng Y, Cheng H, Zhou R. A novel testisenriched gene Spata33 is expressed during spermatogenesis. PLoS ONE. 2013;8:e67882.

53. Monsef L, Borjian Boroujeni P, Totonchi M, Sabbaghian M, Mohseni Meybodi A. Gene alterations and expression spectrum of SPATA33 in nonobstructive azoospermic Iranian men. Mol Reprod Dev. 2018;85:760-7.

54. Yang Y, Klionsky DJ. Autophagy and disease: unanswered questions. Cell Death Differ. 2020;27:858-71.

55. Morishita H, Mizushima N. Diverse cellular roles of autophagy. Annu Rev Cell Dev Biol. 2019;35:453-75.

56. Sheng Y, Song Y, Li Z, Wang Y, Lin H, Cheng H, et al. RAB37 interacts directly with ATG5 and promotes autophagosome formation via regulating ATG5-12-16 complex assembly. Cell Death Differ. 2018;25:918-34.

57. Song Y, Shang D, Cheng H, Zhou R. The small GTPase RAB37 functions as an organizer for autophagosome biogenesis. Autophagy.2018;14:727-9.

58. Gao H, Khawar MB, Li W. Essential role of autophagy in resource allocation during sexual reproduction. Autophagy. 2020;16:18-27.

59. Sanjana NE, Shalem O, Zhang F. Improved vectors and genomewide libraries for CRISPR screening. Nat Methods. 2014;11:783-4.

60. Shalem O, Sanjana NE, Hartenian E, Shi X, Scott DA, Mikkelson $\mathrm{T}$, et al. Genome-scale CRISPR-Cas9 knockout screening in human cells. Science.2014;343:84-7.

61. Yuan J, Zhang Y, Sheng Y, Fu X, Cheng H, Zhou R. MYBL2 guides autophagy suppressor VDAC2 in the developing ovary to inhibit autophagy through a complex of VDAC2-BECN1BCL2L1 in mammals. Autophagy. 2015;11:1081-98.

62. Chen K, Huang C, Yuan J, Cheng H, Zhou R. Long-term artificial selection reveals a role of TCTP in autophagy in mammalian cells. Mol Biol Evol. 2014;31:2194-211. 